\title{
On the Appearance of Thresholds in the Dynamical Model of Star Formation
}

\author{
Bruce G. Elmegreen \\ IBM T. J. Watson Research Center, 1101 Kitchawan Road, Yorktown Heights, New York \\ 10598 USA \\ bge@us.ibm.com
}

\begin{abstract}
The Kennicutt-Schmidt (KS) relationship between the surface density of the star formation rate (SFR) and the gas surface density has three distinct power laws that may result from one model in which gas collapses at a fixed fraction of the dynamical rate. The power law slope is 1 when the observed gas has a characteristic density for detection, 1.5 for total gas when the thickness is about constant as in the main disks of galaxies, and 2 for total gas when the thickness is regulated by self-gravity and the velocity dispersion is about constant, as in the outer parts of spirals, dwarf irregulars, and giant molecular clouds. The observed scaling of the star formation efficiency (SFR per unit $\mathrm{CO}$ ) with the dense gas fraction $(\mathrm{HCN} / \mathrm{CO})$ is derived from the KS relationship when one tracer $(\mathrm{HCN})$ is on the linear part and the other $(\mathrm{CO})$ is on the 1.5 part. Observations of a threshold density or column density with a constant SFR per unit gas mass above the threshold are proposed to be selection effects, as are observations of star formation in only the dense parts of clouds. The model allows a derivation of all three KS relations using the probability distribution function of density with no thresholds for star formation. Failed galaxies and systems with sub-KS SFRs are predicted to have gas that is dominated by an equilibrium warm phase where the thermal Jeans length exceeds the Toomre length. A squared relation is predicted for molecular gas-dominated young galaxies.
\end{abstract}

Subject headings: stars: formation - ISM: molecules — Galaxy: local interstellar matter — galaxies: ISM — galaxies: star formation

\section{Introduction}

A correlation between surface density of star formation, $\Sigma_{\mathrm{SFR}}$, and surface density of gas, $\Sigma_{\text {gas }}$, is observed in local galaxies on scales larger than several hundred parsecs (e.g., 
Kennicutt et al. 2007) and it is observed for whole galaxies over a wide range of redshifts (Daddi et al. 2010; Genzel et al. 2010, see review in Kennicutt \& Evans 2012). This correlation is typically a power law in the main parts of spiral galaxy disks with a fall-off in the outer parts (Kennicutt 1989) that is attributed to a decreasing relative abundance of cool and molecular gas (e.g., Krumholz 2013) with star formation following the molecules (Schruba et al. 2011). The value of the power law slope is consistently around unity for CO in normal galaxies (Wong \& Blitz 2002; Bigiel et al. 2008; Leroy et al. 2008) and for dense gas tracers like HCN (Gao \& Solomon 2004; Wu et al. 2005) and around 1.4 for total gas in the main parts of galaxy disks (Kennicutt 1998). It is steeper in the outer regions of spiral galaxies (Bigiel et al. 2010) and in dwarf irregular galaxies (Roychowdhury et al. 2009; Bolatto et al. 2011; Elmegreen \& Hunter 2015) with a slope of around 2.

The origin of these correlations has been addressed analytically in many previous studies (e.g. Krumholz \& McKee 2005; Krumholz et al. 2009, 2012; Krumholz 2013; Ostriker et al. 2010; Hennebelle \& Chabrier 2011; Padoan \& Nordlund 2011; Renaud et al. 2012; Padoan et al. 2014) and also shown to follow from numerical simulations (e.g., Li et al. 2005; Padoan et al. 2012; Federrath \& Klessen 2012; Kim et al. 2013; Kim \& Ostriker 2015; Hu et al. 2016; Murante et al. 2015; Semenov et al. 2016; Hopkins, et al. 2017). The basic ingredients are gaseous selfgravity, turbulence and cooling, with cloud geometry and feedback determining the gas scale height and limiting the fraction of gas that gets into stars.

A recent development is the inference that star formation not only occurs in dense gas, as long believed, but that the rate of star formation per unit gas mass is independent of the volume density of this gas (e.g., Evans et al. 2014). This inference follows from the linear correlation between $\Sigma_{\mathrm{SFR}}$ and the surface density of dense gas tracers like HCN (Gao \& Solomon 2004; Wu et al. 2005), and from a linear correlation between the total star formation rate (SFR) in molecular clouds and the mass of gas above some extinction threshold, typically $\sim 8$ visual magnitudes (e.g., Lada et al. 2010). This independence from volume density is contrary to expectations based on gravitational processes, which would imply a local collapse rate proportional to the square root of the local density on all scales where the gas motions are supersonic. The purpose of this paper is to question this inference about dense gas and to present an alternative interpretation in which the apparent threshold is the result of one or more selection effects.

The existence of a threshold for any aspect of star formation should be questioned in general. Conceivably, star formation could result from a continuous collapse of interstellar gas, mitigated somewhat by magnetic fields and stellar feedback, all the way from the low density atomic medium (e.g., Michalowski et al. 2015), which is unstable on scales less than the Toomre length (Sect. 7), down to the dense molecular medium and into stars. This 
is contrary to most theories and simulations that assume a threshold density either in an integral over the probability distribution function (PDF) of gas to derive the SFR from first principles (Elmegreen 2002; Kravtsov et al. 2003; Krumholz \& McKee 2005) or from numerical constraints which require a threshold density to convert gas particles into star particles (e.g., Schaye et al. 2015; Vogelsberger et al. 2014). We suggest here that all of the star formation correlations observed in galaxies could follow from a single model of pervasive collapse with no thresholds. We show this in two ways, first with the common dynamical model in which the star formation rate depends on the gas mass divided by the free fall time, and then using integrals over PDFs for star formation rate, total gas, molecular gas, and dynamical time, the latter being used as an independent way to get the molecular gas.

There are several implications of this proposal. First, molecular hydrogen cannot be a pre-requisite for star formation, but a result of the high densities that happen anyway during gas collapse (see also Glover \& Clark 2012; Krumholz 2012). This implication changes the basic equation for star formation (e.g., Krumholz et al. 2012) by removing a term proportional to the molecular fraction: collapse begins in the atomic gas at a large-scale rate that depends primarily the average midplane density, molecular or not. Second, much of the ISM should have the density structure expected for collapse, namely a high-density tail of the density PDF that is a power law. Such power laws are observed in giant molecular clouds (GMCs, e.g., Kainulainen et al. 2011, see other references in Section 3.1), where collapse might be expected anyway, but also in the whole-galaxy PDF for molecules in M33 (Druard et al. 2014). Such pervasive power law structure is the main expectation of the present model and more observations on a galactic scale would be interesting. Third, the gas consumption time varies considerably from place to place, depending on the average gas density and therefore position in a galaxy or type of galaxy, and is not constant for gas above a threshold density or column density as currently inferred from dense gas observations. Dense gas can be stable in high pressure environments and unstable in low pressure environments (Sect. 3.1).

Threshold densities for star formation have also been questioned elsewhere. Gutermuth et al. (2011) found no thresholds for star formation in observations of the MonR2 and Ophiuchus clouds. Lada et al. (2013) and Burkert \& Hartmann (2013) showed that a threshold for SFR inside molecular clouds may appear because of the combination of a uniformly increasing SFR per unit area with gas surface density, i.e., a Schmidt relation with a slope of $\sim 2$ (Sect. 2.5), and a cloud area that varies inversely with surface density. Parmentier (2017) explained the apparently constant star formation rate per unit dense gas mass inside molecular clouds by showing that the total rate integrated over a cloud with an isothermal density profile is proportional to the dense mass in the core of the cloud, even though a significant fraction of the stars form outside the core without an actual density threshold. She also found, for a 
fixed cloud radius, that the SFR transitions from a linear dense mass proportionality when the density at the edge of the cloud is less than the threshold for molecule detection, to a 1.5 power of dense gas mass at a higher mass because of the extra square root dependence on density for the collapse rate. Hopkins, et al. (2017) show that the value of a density threshold used for numerical convenience in a simulation does not affect the star formation rate on large scales as long as the self-gravitating regions are resolved. The present study agrees with the conclusions of these others but differs in detail by deriving the observed KS relations and dense-gas correlations in two ways from a purely dynamical model of pervasive interstellar collapse.

We begin by summarizing the three distinct Kennicutt-Schmidt relations in Section 2 and then we derive them using the dynamical model without thresholds or pre-requisites for molecule formation. A second derivation of the three KS laws in Section 3 uses integrals over the density PDFs, again without thresholds for star formation. Unlike other studies, we use a convolution PDF that includes a power law part at high density. The impact of a column density threshold for the detection of molecules is in Section 4, and an explanation for the appearance of threshold densities and column densities for star formation is in Section 5, A related explanation for the constant star formation rate per unit dense gas mass is in Section 6. Following a discussion (Sect. 7) of how this dynamical model fits into a large-scale picture of interstellar evolution and star formation, the conclusions are in Section 8 ,

\section{Multiple KS Relationships}

\subsection{Three Power Laws}

Most studies of the relationship between $\Sigma_{\text {SFR }}$ and the gas surface density have concentrated on the main disks of spiral galaxies, where a difference in slope is observed for total gas and molecular gas (Kennicutt 1998, Bigiel et al. 2008, see review in Kennicutt \& Evans 2012). In the outer parts of spirals and in dIrrs, a third and steeper slope appears. The transition to this steeper slope occurs where two things happen simultaneously: the average gas column density becomes less than the value needed to shield a molecular cloud (e.g., $\sim 10 M_{\odot} \mathrm{pc}^{-2}$; see Krumholz et al. 2009), and the gas mass begins to dominate the stellar mass, leading to a flare in the total gas thickness as the surface density decreases with an approximately constant velocity dispersion (Olling 1996; Levine et al. 2006). These two things leave an ambiguity in what drives the steeper slope: is it the sudden lack of molecules (Krumholz 2013) or the sudden drop of midplane density in the flare (Barnes et al. 2012; Elmegreen 2015)? 
Here we take the viewpoint that a single dynamical law produces the three observed KS relationships regardless of the pre-existence of molecules with the difference between them the result of primarily two things: the degree of self-gravity in the gas, which determines the relation between the density and the column density, and the ratio of the average density to the characteristic density for radiation of the tracer used to observe it.

To distinguish between these three KS relationships, we group them according to their power laws:

(KS-1a) $\Sigma_{\text {SFR }}$ on galactic and sub-galactic scales increases approximately linearly with the surface density of molecular tracers, such as CO in normal spirals at low density or HCN at high density (Wong \& Blitz 2002; Gao \& Solomon 2004; Wu et al. 2005; Bigiel et al. 2008; Leroy et al. 2008; Heiderman et al. 2010; Bigiel et al. 2011; Wang et al. 2011; Schruba et al. 2011; Bolatto et al. 2011; Leroy et al. 2013; Zhang et al. 2014; Liu et al. 2015; Chen et al. 2017);

(KS-1b) $\Sigma_{\text {SFR }}$ for local molecular clouds scales approximately linearly with the dense gas surface density determined from extinction or FIR emission (Vutisalchavakul et al. 2014);

(KS-1c) The total star formation rate in a molecular cloud scales about linearly with the mass of dense gas (Wu et al. 2010; Lada et al. 2010, 2012; Evans et al. 2014; Vutisalchavakul et al. 2016; Shimajiri et al. 2017);

(KS-1.5) the star formation rate surface density, $\Sigma_{\mathrm{SFR}}$, scales with the total gas surface density, atomic plus molecular, to a power of approximately 1.5 (e.g., Buat et al. 1989; Kennicutt 1989; Tenjes, P.; Haud, U. 1991; Kennicutt 1998; Liu et al. 2015; Calzetti et al. 2017);

(KS-2a) $\Sigma_{\text {SFR }}$ scales approximately with the square of the total gas surface density in the outer regions of spiral galaxies and in dwarf irregular galaxies (Rovchowdhurv et al. 2009; Bigiel et al. 2010; Bolatto et al. 2011; Elmegreen \& Hunter 2015; Elmegreen 2015, hereafter Paper I). Teich et al. 2016 also found a slope of 2 for low-mass galaxies, but only on large scales where stochastic variations were smallest;

(KS-2b) $\Sigma_{\text {SFR }}$ scales with the gas surface density in individual molecular clouds (not just the dense gas) to a power that is approximately 2 (Heiderman et al. 2010; Gutermuth et al. 2011; Harvev et al. 2013; Lada et al. 2013; Evans et al. 2014; Willis et al. 2015; Nguven-Luong et al. 2016; Retes-Romero et al. 2017; Lada et al. 2017).

There is an offset from the KS-1.5 relationship for some ULIRGs (Greve et al. 2005; Daddi et al. 2010; Genzel et al. 2010; Garcia-Burillo et al. 2012), but that is not viewed here as physically distinct from KS-1.5 but as a manifestation of either higher densities from 
galactic-scale shocks (Juneau et al. 2009; Renaud et al. 2014; Kepley et al. 2016), a changing molecular conversion factor (Narayanan et al. 2012), or large corrections to the apparent gas mass (Scoville, et al. 2016).

These relationships follow from the same physical law if we consider that gas can be observed with different tracers and that it can have different relationships between the line of sight depth and the surface density. This law is the commonly assumed dynamical model, which is three-dimensional and written in terms of density, $\rho$, as

$$
\rho_{\mathrm{SFR}}=\epsilon_{\mathrm{ff}} \rho / t_{\mathrm{ff}}
$$

where

$$
t_{\text {ff }}=(32 G \rho /[3 \pi])^{-1 / 2}
$$

is the free fall time and $\epsilon_{\mathrm{ff}}$ is an approximately constant efficiency per unit free fall time (e.g., Larson 1969; Madore 1977; Elmegreen 1991, 2002; Krumholz \& McKee 2005). In the present model, there is no term like the molecular fraction, $f_{\mathrm{H} 2}$, in Krumholz et al. (2012) because molecules, molecular clouds, HCN regions etc., are all viewed as incidental and not causal, so they can be ignored in the equation for star formation, which is primarily a dynamical process. Observations of $\epsilon_{\mathrm{ff}}$ for individual star-forming regions suggest a range of values consistent with time-variability (Lee et al. 2016); numerical simulations get a range for $\epsilon_{\mathrm{ff}}$ too (Semenov et al. 2016).

A summary of analytical models that reproduce these relationships follows. Some are newly derived and all follow from equation (1) without thresholds.

\subsection{KS-1.5: Star formation for total gas in main spiral galaxy disks}

KS-1.5 is the standard relation for star formation in galaxies (Buat et al. 1989; Kennicutt 1989, 1998) so we begin with that here. It follows from equation (1) if the observed disk region has an approximately constant scale height, $H$, as observed for CO in the Milky Way (Heyer \& Dame 2015). Then $\rho=\Sigma / 2 H$ and

$$
\Sigma_{\mathrm{SFR}}=\epsilon_{\mathrm{ff}}(16 G /[3 \pi H])^{1 / 2} \Sigma_{\text {gas }}^{3 / 2} .
$$

With typical $H=100 \mathrm{pc}$ and $\epsilon_{\mathrm{ff}}=0.01$, this becomes

$$
\frac{\Sigma_{\mathrm{SFR}}}{M_{\odot} \mathrm{pc}^{-2} \mathrm{Myr}^{-1}}=8.8 \times 10^{-5}\left(\frac{\Sigma_{\mathrm{gas}}}{M_{\odot} \mathrm{pc}^{-2}}\right)^{1.5} .
$$

This result was shown in Paper I to agree in both slope and intercept with the observations in Kennicutt \& Evans (2012), which are for average star formation rates in the main disks 
of spiral galaxies (the average rate per unit area in a galaxy is a good reflection of the local rate; Elmegreen 2007a).

The approximately constant $H$ requires a separate model with a more complete theory of interstellar processes (e.g., Kim \& Ostriker 2015). For example, the squared velocity dispersion of the gas, $\sigma^{2}$, should be proportional to the total mass surface density in the gas layer because $H=\sigma^{2} /\left(\pi G \Sigma_{\text {tot }}\right)$ is then constant. Such a relation may be a consequence of the KS-1.5 relation if we consider that the energy density decay rate per unit area in the gas, $0.5 \Sigma_{\text {gas }} \sigma^{3} / H$ (for dissipation in a crossing time $H / \sigma$; Mac Low et al. 1998; Stone et al. 1998), is proportional to the product of the gas surface density and the SFR, $\propto \Sigma_{\text {gas }} \Sigma_{\mathrm{SFR}}$. Then $\Sigma_{\text {gas }} \sigma^{3} / H=\pi G \Sigma_{\text {gas }} \Sigma_{\text {tot }} \sigma \propto \Sigma_{\text {gas }} \Sigma_{\text {SFR }} \propto \Sigma_{\text {gas }}^{2.5}$ so that $\sigma \propto \Sigma_{\text {gas }}^{0.5}\left(\Sigma_{\text {gas }} / \Sigma_{\text {tot }}\right)$ and $H \sim$ $\left(\Sigma_{\text {gas }} / \Sigma_{\text {tot }}\right)^{3}$. This ratio for $H$ is about constant in the main disks of spiral galaxies where both stars and gas have the same exponential scale lengths.

\subsection{KS-1a: Star formation in molecular gas at the characteristic density for emission}

\subsubsection{Derivation from Analytical Theory}

KS-1a follows for a constant effective density for emission, $\rho_{\text {mol }}$ (Evans 1999; Shirley 2015; Jiménez-Donaire et al. 2017; Leroy et al. 2017a), that is much larger than the average interstellar density, $\rho_{\text {gas }}$. Then equation (1) converts to

$$
\Sigma_{\mathrm{SFR}}=\epsilon_{\mathrm{ff}} \Sigma_{\mathrm{mol}} / t_{\mathrm{ff}, \mathrm{mol}}
$$

for constant $t_{\mathrm{ff}, \mathrm{mol}}$ equal to the free fall time at the characteristic density for emission by the molecule (which could be $\mathrm{CO}, \mathrm{HCN}$, or some other tracer of a particular phase of gas). Here we have set the fraction of the interstellar medium in the molecular phase equal to the fraction of the time spent as molecules, based on the local dynamical time (Paper I).

$$
f_{\mathrm{mol}}=\frac{\rho_{\mathrm{mol}}^{-0.5}}{\rho_{\mathrm{mol}}^{-0.5}+\rho_{\mathrm{gas}}^{-0.5}} \sim\left(\frac{\rho_{\mathrm{gas}}}{\rho_{\mathrm{mol}}}\right)^{0.5}
$$

(the approximation is for partially molecular regions, $\rho_{\text {gas }}<<\rho_{\text {mol }}$ ). This expression assumes that both the cloud formation time before star formation and the cloud break-up time after star formation are proportional to the local dynamical times, in agreement with numerical simulations of a supernova-agitated interstellar medium (Padoan et al. 2016a). Then

$$
\Sigma_{\mathrm{mol}}=f_{\mathrm{mol}} \Sigma_{\mathrm{gas}}
$$

and equation (5) follows from equation (1). 


\subsubsection{KS-1a in Comparison to Observations}

To evaluate equation (5) in case KS-1a, we consider that CO appears in local clouds at about 1.5 magnitude of visual extinction (Pineda et al. 2008), which corresponds to $\sim 30 M_{\odot}$ $\mathrm{pc}^{-2}$ of column density. For a typical large cloud near this extinction threshold with a size of $\sim 30 \mathrm{pc}$, the $3 \mathrm{D}$ density is $\sim 17 \mathrm{H}_{2} \mathrm{~cm}^{-3}$ and for this density $t_{\mathrm{ff}, \text { mol }}=8.0$ Myr. With $\epsilon=0.01$ again, equation (5) becomes

$$
\frac{\Sigma_{\mathrm{SFR}}}{M_{\odot} \mathrm{pc}^{-2} \mathrm{Myr}^{-1}}=1.2 \times 10^{-3}\left(\frac{\Sigma_{\mathrm{mol}, \mathrm{CO}}}{M_{\odot} \mathrm{pc}^{-2}}\right)
$$

giving a consumption time of 0.80 Gyr. This molecular consumption time is too short by a factor of $\sim 2$ compared to star formation rates on a large scale (e.g., Bigiel et al. 2008; Leroy et al. 2008), suggesting that $\epsilon_{\mathrm{ff}} / t_{\mathrm{ff}}$ should be lower by this factor. For example, Leroy et al. (2017b) find $\epsilon_{\mathrm{ff}} \sim 0.003$ on $40 \mathrm{pc}$ scales in M51 and discuss how $\epsilon_{\mathrm{ff}}$ is often observed to be lower than 0.01. Murray (2011) suggest $\epsilon_{\mathrm{ff}} \sim 0.006$ on average in the Milky Way.

Leroy et al. (2017b) considered star formation relationships for CO observations at 40 pc resolution in M51. They found that the molecular depletion time, which is $\Sigma_{\text {mol }} / \Sigma_{\mathrm{SFR}}=$ $t_{\mathrm{ff}, \mathrm{mol}} / \epsilon_{\mathrm{ff}}$ in our notation, is approximately constant instead of their expected $\Sigma_{\mathrm{mol}}^{-0.5}$ for dynamical star formation at fixed $\epsilon_{\mathrm{ff}}$. However, this depletion time should be constant if the average density for observations of $\mathrm{CO}$ is constant, as above, because that gives the linear molecular relation, KS-1a. The stated expectation was that the density used for the dynamical time would be proportional to the average density in the 40 pc region, but that is not the case if the average $\mathrm{CO}$ density in the resolution element is less than the characteristic density for $\mathrm{CO}$ emission. The average density comes from the summed mass of the CO clouds in the $40 \mathrm{pc}$ region, but each cloud could have about the same characteristic density for CO emission and the same $t_{\mathrm{ff} \text {,mol }}$. The best correlation they found was with the virial parameter, $5 R \sigma^{2} /(G M)$ for $R=40 \mathrm{pc}$ and mass $M$ inside the region. They determined that the depletion time scales with the virial parameter to a power of $\sim 0.9$. At the same time, Leroy et al. (2017b) found that $\epsilon_{\mathrm{ff}}$ is nearly independent of the virial parameter. These two results imply, for the dynamical model, that the average density per molecular cloud, which occurs inside $t_{\mathrm{ff}, \mathrm{mol}}$, depends on the average virial parameter measured on the scale of $40 \mathrm{pc}$.

In the case of a dense molecular tracer, like $\mathrm{HCN}$ or $\mathrm{HCO}^{+}$, the characteristic density of observation is $\sim 3 \times 10^{4} \mathrm{~cm}^{-3}$ in equation (5), giving $t_{\mathrm{ff}, \text { mol }}=0.19 \mathrm{Myr}$ and with $\epsilon=0.01$,

$$
\frac{\Sigma_{\mathrm{SFR}}}{M_{\odot} \mathrm{pc}^{-2} \mathrm{Myr}^{-1}}=0.052\left(\frac{\Sigma_{\mathrm{mol}, \mathrm{HCN}}}{M_{\odot} \mathrm{pc}^{-2}}\right)
$$

The average observed coefficient is slightly lower than 0.052 , i.e., more like 0.02 (Sect. 6), 
so $\epsilon_{\mathrm{ff}}$ is proportionally lower or the characteristic density for emission is slightly higher. The result is close enough to the observation to support the general model, given the uncertainties in density, star formation rate, and dense mass, plus the approximate nature of the model itself.

At high interstellar density (which usually corresponds to high $\Sigma_{\text {gas }}$ ), $\rho_{\text {gas }} \gtrsim \rho_{\text {mol }}$ and $f_{\text {mol }} \sim 1$, in which case equation (3) applies with $\Sigma_{\text {mol }} \sim \Sigma_{\text {gas }}$. Thus molecular emission has a 1.5 power law at high $\rho_{\text {gas }}$ (if $H$ is still about constant) and a linear law at interstellar densities below the effective density for emission where $f_{\text {mol }}<1$ (e.g., Kennicutt 1998; Krumholz et al. 2012). For example, Gowardhan et al. (2017) got a slope of $1.41 \pm 0.10$ for CO emission in ULIRGS and high redshift galaxies where the density is large $\left(\rho_{\text {gas }} \gtrsim \rho_{\text {mol }}\right.$ for $\mathrm{CO}$ ), and they got a slope closer to unity, $1.11 \pm 0.05$, for the dense gas tracer HCN in the same galaxies, presumably because $\rho_{\text {gas }} \lesssim \rho_{\text {mol }}$ and $f_{\text {mol }}<1$ for HCN in these galaxies. (For more discussion on this point, see Krumholz \& Thompson 2007; Naravanan et al. 2008; Burkert \& Hartmann 2013; Elmegreen 2015).

\subsubsection{The Relationship between Star Formation Efficiency and Dense Gas Fraction}

An important correlation appears for average interstellar densities that are between the characteristic densities for $\mathrm{CO}$ and HCN observations (or any other low and high density tracers). Above the $\mathrm{CO}$ density, $\mathrm{CO}$ tracks the total interstellar density fairly well because $f_{\text {mol,CO }} \sim 1$ and then $\Sigma_{\mathrm{SFR}} \propto \Sigma_{\mathrm{CO}}^{1.5}$ for a constant thickness galaxy, as mentioned above. Below the HCN density, $f_{\text {mol,HCN }}<1$ for the average interstellar medium and then the linear law appears: $\Sigma_{\mathrm{SFR}} \propto \Sigma_{\mathrm{HCN}}$. Thus the ratio of HCN to CO, which is viewed as the "dense gas fraction," increases with the star formation rate,

$$
f_{\text {dense }}=\frac{\Sigma_{\mathrm{HCN}}}{\Sigma_{\mathrm{CO}}} \propto \Sigma_{\mathrm{SFR}}^{1 / 3} \propto \Sigma_{\mathrm{CO}}^{1 / 2}
$$

Similarly, the "star formation efficiency", measured as the ratio $\Sigma_{\mathrm{SFR}} / \Sigma_{\mathrm{CO}}$, should scale linearly with $f_{\text {dense: }}$

$$
\mathrm{SFE}=\frac{\Sigma_{\mathrm{SFR}}}{\Sigma_{\mathrm{CO}}} \approx \frac{\Sigma_{\mathrm{CO}}^{3 / 2}}{\Sigma_{\mathrm{CO}}}=\Sigma_{\mathrm{CO}}^{1 / 2} \approx \Sigma_{\mathrm{SFR}}^{1 / 3} \approx f_{\text {dense }}
$$

These correlations are consistent with observations of star-forming galaxies and ULIRGs in Usero et al. (2015) and elsewhere. Similarly, Gao \& Solomon (2004) observed $L_{\mathrm{HCN}} / L_{\mathrm{CO}} \propto$ $L_{\mathrm{CO}}^{0.38}$ which is similar to our prediction of a power of $\sim 0.5$ in this middle-density regime at constant $H$, and they observe $L_{\mathrm{IR}} / L_{\mathrm{CO}} \propto\left(L_{\mathrm{HCN}} / L_{\mathrm{CO}}\right)^{1.24}$, which is similar to our predicted power of 1 . Sections 4 and 6 return to discuss star formation in dense gas, including sublinear relations between SFR and HCN which are not considered above. 


\subsection{KS-2a: Star Formation in Total Gas for dIrrs and Outer Spiral Galaxy Disks}

KS-2 follows when the line-of-sight thickness of the region is in pressure equilibrium with gas self-gravity for the observed gas column density. This should be the case in the gas-dominated parts of galaxy disks. We set the disk scale height $H=\sigma^{2} / \pi G \Sigma$ and then derive (Paper I)

$$
\Sigma_{\mathrm{SFR}}=(4 / \sqrt{3}) \epsilon_{\mathrm{ff}} G \Sigma_{\text {gas }}^{2} / \sigma .
$$

With a constant velocity dispersion $\sigma=6 \mathrm{~km} \mathrm{~s}^{-1}$ as typically observed in dwarf irregulars and outer spiral disks, and for $\epsilon_{\mathrm{ff}}=0.01$,

$$
\frac{\Sigma_{\mathrm{SFR}}}{M_{\odot} \mathrm{pc}^{-2} \mathrm{Myr}^{-1}}=1.7 \times 10^{-5}\left(\frac{\Sigma_{\text {gas }}}{M_{\odot} \mathrm{pc}^{-2}}\right)^{2} .
$$

This relation was shown in Paper I to agree with observations of the outer parts of spiral disks and dwarf irregular galaxies. The main reason for the steepening of the slope is the increase in scale height with radius, i.e., the disk flare in a galaxy. That increase drops the midplane gas density faster than the surface density so the dynamical rate at the midplane

density drops more quickly too. A disk flare was also present in the Krumholz (2013) model although not mentioned explicitly.

These regions of low surface brightness are also where the metallicity tends to be low (Rosales-Ortega et al. 2012; Bresolin \& Kennicutt 2015), but the drop in $\Sigma_{\text {SFR }}$ is probably not from an inability to make $\mathrm{H}_{2}$ on dust. This is because the star formation relation in this regime is the same for a wide range in metallicities, i.e., comparing outer spiral disks where the metallicity is slightly below solar to dwarf irregular galaxies, where the metallicity is $\sim 10 \%$ solar (Roychowdhury et al. 2015; Jameson et al. 2016). The squared dependence of $\Sigma_{\mathrm{SFR}}$ on $\Sigma_{\text {gas }}$ is also not from a drop in molecular fraction with decreasing density because the density dependence of the molecular fraction for conventional theory (Krumholz et al. 2009 ) is much steeper than the observed decrease in $\Sigma_{\mathrm{SFR}}$ with gas density in galaxies (Elmegreen \& Hunter 2015).

Ostriker \& Shetty (2011) derived a squared KS relation on a galactic scale by assuming that the interstellar pressure is proportional to $\Sigma_{\mathrm{SFR}}$ through momentum injected by supernovae, and that this pressure is also proportional to $\Sigma_{\text {gas }}^{2}$ as in an equilibrium galaxy disk. The application of supernova regulation in outer spiral disks and dIrr galaxies is not clear though, considering the very low star formation rate and pressure there. For example, in an exponential disk, the surface density of supernovae decreases as $\exp \left(-R / R_{\mathrm{D}}\right)$ for star formation scale length $R_{\mathrm{D}}$, and the midplane gas density decreases as $\rho \propto \exp \left(-2 R / R_{\mathrm{D}}\right)$ considering the outer-disk flare (i.e., $\rho=(\pi / 2) G \Sigma^{2} / \sigma^{2}$ for an equilibrium disk of pure gas 
with a near-constant velocity dispersion $\sigma$ and the same exponential for gas surface density, $\Sigma)$. Considering that the radius at which a supernova remnant merges with the ambient medium scales as $\rho^{-3 / 49}$ (Cioffi et al. 1988), it follows that the volume filling factor of remnants, which is this radius cubed multiplied by the space density of supernovae, decreases with galactocentric radius as

$$
f_{\mathrm{SNR}} \sim e^{-R / R_{\mathrm{D}}} e^{18 R / 49 R_{\mathrm{D}}} \sim e^{-0.63 R / R_{\mathrm{D}}} .
$$

Thus, outer galaxy disks should have relatively sparse stirring by supernovae. A flatter decrease than this for the total gas surface density, e.g., from an extended HI disk, makes this conclusion even stronger.

\subsection{KS-2b: Star Formation on the Molecular Cloud Scale}

KS-2b may follow from the same relationship as KS-2a if it is applied to the interiors of self-gravitating clouds or to whole self-gravitating clouds. We assume a power-law internal density profile $\rho(r)=\rho_{\text {edge }}\left(r_{\text {edge }} / r\right)^{\alpha}$ from some small core radius, $r_{\text {core }}$ to the edge radius $r_{\text {edge }}$ where the density is $\rho_{\text {edge }}$ (an internal profile that explicitly includes $\rho_{\text {core }}$ is in equation (30)). This gives a radius-dependent mass

$$
M(r)=\frac{4 \pi}{3-\alpha} \rho_{\text {edge }} r_{\text {edge }}^{\alpha} r^{3-\alpha}
$$

and surface density $\Sigma(r)=M(r) /\left(\pi r^{2}\right)$. As an approximation, we take the one-dimensional velocity dispersion $\sigma(r)$ from the virial theorem,

$$
3 \int_{0}^{r} \sigma(r)^{2} \rho(r) 4 \pi r^{2} d r=\int_{0}^{r}(G M[r] / r) \rho(r) 4 \pi r^{2} d r
$$

which gives

$$
\sigma(r)^{2}=\frac{4 \pi G}{3(3-\alpha)} \rho_{\text {edge }} r_{\text {edge }}^{\alpha} r^{2-\alpha}=G M(r) /(3 r) .
$$

The internal surface density for the SFR then follows from equation (11), which is also a function of radius,

$$
\Sigma_{\mathrm{SFR}}(r)=\frac{1}{\pi r^{2}} \int_{0}^{r}\left(\frac{\epsilon_{\mathrm{ff}} \rho(r)}{t_{\mathrm{ff}}(r)}\right) 4 \pi r^{2} d r .
$$

For a singular isothermal sphere, $\alpha=2$, the SFR surface density has a logarithmic divergence near the center of the cloud, which requires the use of a core radius,

$$
\Sigma_{\mathrm{SFR}}(r)=\sqrt{8 / 9} \epsilon_{\mathrm{ff}}\left(\frac{G \Sigma(r)^{2}}{\sigma(r)}\right) \ln \left(r / r_{\text {core }}\right) .
$$


For other $\alpha<2$,

$$
\Sigma_{\mathrm{SFR}}(r)=\sqrt{8 / 9}\left(\frac{(3-\alpha)^{1.5}}{3-1.5 \alpha}\right) \epsilon_{\mathrm{ff}}\left(\frac{G \Sigma(r)^{2}}{\sigma(r)}\right) .
$$

We assume for comparison with observations that $\ln \left(r / r_{\text {core }}\right) \sim(3-\alpha)^{1.5} /(3-1.5 \alpha) \sim 2.45$, which follows from the first expression when $\alpha=2$ if $r / r_{\text {core }}=11.6$ and from the latter expression if $\alpha=1.5$, which are two cases considered also in Section 3. Then

$$
\Sigma_{\mathrm{SFR}}(r) \approx 2.31 \epsilon_{\mathrm{ff}} G \Sigma(r)^{2} / \sigma(r) .
$$

These results were written to resemble equation (12) in form.

Table 2 in Gutermuth et al. (2011) gave fits for the $\Sigma_{\mathrm{SFR}}-\Sigma_{\text {gas }}$ relation for 8 local molecular clouds. Averaging their coefficients in the log and averaging their powers of $\Sigma_{\text {gas }}$, we get from their observations

$$
\frac{\Sigma_{\mathrm{SFR}}}{M_{\odot} \mathrm{pc}^{-2} \mathrm{Myr}^{-1}} \approx 3.6 \times 10^{-4}\left(\frac{\Sigma_{\mathrm{gas}}}{M_{\odot} \mathrm{pc}^{-2}}\right)^{2.2}
$$

This observation agrees with equation (21) at $\Sigma_{\text {gas }}=100 M_{\odot} \mathrm{pc}^{-2}$ and $\sigma=1 \mathrm{~km} \mathrm{~s}^{-1}$ if $\epsilon_{\mathrm{ff}}=0.09$. This efficiency inside molecular clouds is factor of $\sim 9$ larger than it is on a galactic scale but perhaps this excess is reasonable if the galactic scale contains $\mathrm{CO}$ gas that is not connected with SF.

Lada et al. (2013) fitted the star formation rates in three local clouds, Orion A, Taurus and California Nebula,

$$
\frac{\Sigma_{\mathrm{SFR}}}{M_{\odot} \mathrm{pc}^{-2} \mathrm{Myr}^{-1}} \approx 4.6 \times 10^{-5}\left(\frac{\Sigma_{\text {gas }}}{M_{\odot} \mathrm{pc}^{-2}}\right)^{2.0},
$$

which is a factor of $\sim 8$ lower than in equation (22). This would translate to $\epsilon_{\mathrm{ff}}=0.005$ in equation (21) for $\sigma \sim 1 \mathrm{~km} \mathrm{~s}^{-1}$. These studies determine the local star formation rates from counts of young stars, which involve assumptions about stellar ages and masses, and there could also be stochastic effects for low counts. Lada et al. (2013) note that the relation is steeper for Orion $\mathrm{B}$, where the slope is 3.3. The real relation should scale with $\Sigma_{\text {gas }}^{2} / \sigma$, however, and $\sigma$ may vary with $\Sigma_{\text {gas }}$ (see below).

Gutermuth et al. (2011) considered a reason for their molecular cloud relation that is somewhat like ours, deriving a KS-2b relation based on counting the areal density of thermal Jeans mass objects in a thin self-gravitating cloud. Equation (21) is more general and shows directly the connection between the molecular cloud relation and the galactic relation for gas-dominated regions (KS-2a). Parmentier \& Pfalzner (2013) derived approximately the same squared star formation law for molecular clouds as in equation (21) using the area 
integral over a collapse-model density profile (see also Parmentier et al. 2011). They applied it to the formation of star clusters.

Other observations of the KS relation on molecular cloud scales are in Heiderman et al. (2010), Harvev et al. (2013), Lada et al. (2013), Willis et al. (2015), Heyer et al. (2016), Nguyen-Luong et al. (2016), Retes-Romero et al. (2017) and Lada et al. (2017), who all found a power index of $\Sigma_{\text {gas }}$ close to 2 (or sometimes larger) and a similar factor of $\sim 20$ efficiency compared to the galactic-scale KS law.

Evans et al. (2014) considered young stellar objects in 25 local clouds using the c2d and Gould Belt Spitzer legacy program (Evans et al. 2003; Dunham et al. 2013). They plotted the KS relation for these clouds with 3D density instead of column density, using the same thickness for the star formation rate and the gas, so it is essentially the same as plotting surface densities. They got a slope of $2.02 \pm 0.07$ and suggested that this slope is inconsistent with the dynamical star formation model. That inconsistency is only in comparison to KS1.5, however, with its slope of 1.5 for main galaxy disks.

Equation (21) should also apply to whole molecular clouds if the radius is taken to be the cloud radius so the average surface density and cloud dispersion are used. For the Evans et al. (2014) data with tabulated values of $\Sigma_{\mathrm{SFR}}, \Sigma_{\text {gas }}$ and $\sigma$, we derive a proportionality constant $\epsilon_{\mathrm{ff}}=0.016$ for equation (21). Another survey of star-forming complexes was made for the Large Magellanic Cloud (Ochsendorf et al. 2017). The average value of $\Sigma_{\mathrm{SFR}} /\left(\Sigma_{\mathrm{gas}}^{2} / \sigma\right)$ for that implies $\epsilon_{\mathrm{ff}}=0.04$. These values are reasonably consistent with other values of $\epsilon_{\mathrm{ff}}$ discussed in this paper, considering the difficulty in defining the local star formation rate in a molecular cloud.

Heyer et al. (2016) saw no KS relationship for young stellar objects in Milky Way molecular clouds because the range in $\Sigma_{\text {gas }}$ was too small (their figure 10a), but they compared it only with the galactic $\sim 1.5$ law and the galactic linear molecular law. The plotted points actually show a steeper relationship on average, consistent with a slope of 2 or 3 . Heyer et al. (2016) did find an approximately linear correlation between $\Sigma_{\mathrm{SFR}}$ and $\Sigma_{\text {gas }} / t_{\mathrm{ff}}$, however, and this is the basic model assumed in equation (21).

Wu et al. (2010) compared the total star formation rates in molecular clouds, $d M_{\text {star }} / d t$, with the local rates in the dynamical model, $\rho_{\text {gas }}^{1.5}$, and got a decreasing relationship in their Figure 35 which they claimed was inconsistent with an expected positive correlation in the dynamical model. However, they should have compared the total rate with the product of the cloud volume times the local rate, which would have introduced an additional term $\left(\Sigma_{\text {gas }} / \rho_{\text {gas }}\right)^{3}$ for cloud volume to be multiplied by the local rate $\rho_{\text {gas }}^{1.5}$. The result would have been proportional to $\Sigma_{\text {gas }}^{3} \rho_{\text {gas }}^{-1.5}$ as in their observed decreasing relation, considering that their 
$\Sigma_{\text {gas }}$ had a narrow range. Thus their result is also consistent with molecular cloud evolution on a self-gravitating timescale. Their explanation for the decreasing relationship is that cloud mass and therefore IR luminosity is correlated inversely with density, as found by Larson (1981), but the KS relationship should be between IR luminosity per unit volume and gas density (or IR luminosity per unit area and gas surface density).

The small-scale mechanism of star formation inside molecular clouds is not addressed by the simple dynamical model of equation (21). Molecular clouds appear to be composed of numerous filaments (André et al. 2010; Molinari et al. 2010) and it might be that collisions between these filaments trigger local star formation (Mvers 2009; Schneider et al. 2012). Parmentier (2017) considered the cloud KS law with filamentary extensions to large radius. The density PDF for filamentary structure has been considered by Myers (2015). If mutual gravity is involved, causing the filaments to collide with each other, then the dynamical model should still apply because it states only that the rate of star formation on a large scale, i.e., averaged over many filaments, is proportional to the rate of mutual gravitational attraction.

\subsection{Other Considerations}

Krumholz et al. (2012) suggested a two-regime model for KS-1.5 using the line-of-sight integrated form of equation (11) where $\Sigma_{\mathrm{SFR}}$ is proportional to $\Sigma_{\text {gas }} / t_{\mathrm{ff}}$. In one regime, the density used for $t_{\mathrm{ff}}$ was the $3 \mathrm{D}$ cloud density of a Jeans-mass cloud with a fixed surface density, $\Sigma_{\mathrm{GMC}}$, and in another regime, the density for $t_{\mathrm{ff}}$ was the average disk value when Toomre $Q=1$. The value of $t_{\mathrm{ff}}$ used for the relationship was the smaller of these two. While this method gave a good fit to the data, we consider that clouds with a fixed $\Sigma_{\mathrm{GMC}}$ are more or less star-forming depending on the local interstellar pressure (see equation 24 below), and that $Q \sim$ constant is not a dependable criterion for interstellar properties (Sect. 7). Still, the utility of a KS law written explicitly in terms of $\Sigma_{\text {gas }} / t_{\mathrm{ff}}$ rather than $\Sigma_{\text {gas }}$ alone, or one written in terms of $\Sigma_{\text {gas }}^{2} / \sigma$ for self-gravitating regions, is that important dynamical processes can be considered when $t_{\mathrm{ff}}$ or $\sigma$ are included in addition to the total available gas for star formation.

KS-1.5 and KS-1 break down on small scales because star formation and cloud evolution are time dependent, and observations on small scales no longer see the average values that are used in the simple theory reviewed here (Schruba et al. 2010; Kruijssen \& Longmore 2014).

KS-1.5, KS-1, and KS-2a also contain another, hidden, relation that is independent of 
star formation and that is the simultaneous radial decrease of both $\Sigma_{\text {SFR }}$ and $\Sigma_{\text {gas }}$ from the exponential profile of a galaxy disk (Bolatto et al. 2017). This dependence stretches out the relation to cover a large range in both quantities. Azimuthal variations from spiral arms also contain an independent relation that stretches out the parameter range. Spiral arms collect and disperse molecular gas and its associated star formation without much of a change in the star formation rate per unit molecular mass (Ragan et al. 2016; Schinnerer et al. 2017). These additional dependencies can be important in some cases. For example, azimuthal variations of $\Sigma_{\text {gas }}$ in dwarf irregular galaxies may not have associated variations in the disk thickness and then KS-1.5 or KS-1a would apply to those variations, while KS-2a still applies in the radial direction as the thickness increases.

The dense gas relations KS-1b and KS-1c do not follow from the dynamical model where cloud evolution is always proportional to the gravitational collapse rate. We suspect KS-1b and KS-1c may be artifacts of observational selection, as discussed in Sections 4, 5, and 6.

\section{Power law Probability Distribution Functions and their Role in the KS Relation}

\subsection{Integrals over the Convolution PDF Function}

The previous section showed how observations of star formation in total gas or in $\mathrm{CO}$ and HCN molecules follow from equation (11) for main and outer galaxy disks, dIrrs, and individual GMCs. Another way to derive the SFR has been to use an integral over the interstellar density PDF above some threshold density, assuming the PDF is a log-normal (Elmegreen 2002; Kravtsov et al. 2003; Krumholz \& McKee 2005). This use of a threshold density assumes that all of the gas above the threshold is involved with the star formation process and is therefore strongly self-gravitating. This cannot be the case for a fixed threshold, however. What matters is the virial parameter, $5 R \sigma^{2} / G M$, for a particular interstellar cloud of radius $R$, velocity dispersion $\sigma$ and mass $M$ (Padoan et al. 2012). This parameter has to be less than about unity for strong self-gravity. That condition is the same as requiring a threshold (minimum) column density that depends only on the ambient pressure $P:$

$$
\Sigma_{\text {Threshold }}=\left(\frac{2 P}{\pi G}\right)^{0.5} .
$$

(given that the pressure in a self-gravitating cloud is $\pi G \Sigma^{2} / 2$ for cloud column density $\Sigma$ ).

In high-pressure regions of galaxies, such as the inner regions where both the stellar and the gas surface densities are high (the pressure scales with the product of these two 
quantities), regions with a certain fixed column density like 8 magnitudes of visual extinction will not be self-gravitating. They will be like the diffuse clouds observed locally where the pressure is low. Similarly, in the far-outer regions of galaxies or in dwarf irregulars and low surface-brightness galaxies, where the pressure is low, clouds with even modest column densities can be self-gravitating if they exceed the threshold given by equation (24) (see also the discussion in Elmegreen 2013). A fixed column density or, correspondingly, a fixed density at a given cloud mass, cannot be part of a universal condition for star formation. Thus we consider here the KS relations using density PDFs without threshold densities, and we use power-law PDFs as recently observed on the cloud scale and possibly larger (e.g. Druard et al. 2014).

Turbulence that randomly compresses and decompresses gas takes the local density on a random walk in the log of the density, and this makes the PDF of density a lognormal (Vázquez-Semadeni 1994; ?). Interstellar gas that has some density structure in addition to local turbulence should have a PDF equal to the convolution of that structure with the log-normal from local turbulence (Elmegreen 2011). This implies that gravitating gas with power-law density gradients in dense cores and filaments should have a powerlaw PDF, as simulated (Klessen 2000; Vázquez-Semadeni et al 2008; Kritsuk et al. 2011; Federrath \& Klessen 2013; Pan et al. 2016) and observed with dust extinction (Froebrich \& Rowles 2010; Kainulainen et al. 2011), dust emission (Schneider et al. 2013; Lombardi et al. 2015; Schneider et al. 2015a., c) and molecular line emission (Schneider et al. 2016). Schneider et al. (2015b) found a power law characteristic of collapse up to $A_{\mathrm{V}} \sim 100 \mathrm{mag}$ and then a flatter power law beyond, which they supposed was from some termination of the collapse. Schneider et al. (2012) determined the PDF of the Rosette molecular cloud and suggested it had an extension to higher density because of compression from the nebula.

In a steady-state, collapse-like motions have a local velocity, $v(r)$, proportional to $(G M(r) / r)^{0.5}$ for mass $M(r)$ inside radius $r$, and these motions produce a density gradient toward the collapse center, $\rho(r)$, that makes the inflow flux, $4 \pi r^{2} v(r) \rho(r)$, approximately constant. The solution to these equations is $\rho \propto r^{-2}$. A singular isothermal sphere in virial equilibrium also has this density profile, while a collapsing envelope onto a core can have a $\rho(r) \propto r^{-3 / 2}$ profile (Shu 1977). Observations of these density gradients in dense cores mapped by sub-mm wave dust emission were in Mueller et al. (2002).

In general, if $\rho \propto r^{-\alpha}$ then the PDF slope is $-3 / \alpha$ (Kritsuk et al. 2011; Elmegreen 2011). This result may be derived for $\rho \propto r^{-\alpha}$ from the expression where density and radius correspond one-to-one, $P_{\mathrm{PDF} .3 \mathrm{D}}(\rho) d \rho=P_{3 \mathrm{D}}(r) d r$, which gives (e.g., see also Federrath \& Klessen 2013; Schneider et al. 2013; Girichidis et al. 2014)

$$
P_{\mathrm{PDF}, 3 \mathrm{D}}(\rho)=P_{3 \mathrm{D}}(r) /(d \rho / d r) .
$$


Considering that the probability of radius $r$ is $P_{3 \mathrm{D}}(r)=4 \pi r^{2}$ and that $d \rho / d r \propto-r^{-\alpha-1}$, this gives $P_{\mathrm{PDF}, 3 \mathrm{D}} \propto r^{\alpha+3} \propto \rho^{-1-3 / \alpha}$ for equal intervals of $\rho$ and $\rho^{-3 / \alpha}$ for equal intervals of $\ln \rho$.

In the same way, the power in the PDF for surface density may be obtained from the one-to-one relation $P_{\mathrm{PDF}, 2 \mathrm{D}}(\Sigma) d \Sigma=P_{2 \mathrm{D}}(b) d b$ for impact parameter $b$. With $\Sigma \propto b^{-\beta}$ and $P_{2 \mathrm{D}} \propto b$, the result is $P_{\mathrm{PDF}, 2 \mathrm{D}} \propto b^{\beta+2} \propto \Sigma^{-1-2 / \beta}$ for equal intervals of $\Sigma$ and $\Sigma^{-2 / \beta}$ for equal intervals of $\ln \Sigma$. For a spherical cloud, $\beta=\alpha-1$, so if $\alpha=2$, then $\beta=1$ and $P_{\mathrm{PDF}, 2 \mathrm{D}} \propto \Sigma^{-2}$ for equal $\ln \Sigma$ intervals. For $\alpha=3 / 2, P_{\mathrm{PDF}, 2 \mathrm{D}} \propto \Sigma^{-4}$ for equal $\ln \Sigma$ intervals.

In a more general situation (Elmegreen 2011), the total 3D PDF may be approximated by the convolution of the average radial density profile, $\rho_{\text {ave }}(r)$, and the local PDF representing fluctuations around this average, $P_{\mathrm{PDF}, \text { local }}$ :

$$
P_{\mathrm{PDF}, 3 \mathrm{D}}(\rho)=\int_{\rho_{\text {ave min }}}^{\rho_{\text {ave max }}} P_{\mathrm{PDF}, \text { local }}\left(\rho \mid \rho_{\text {ave }}\right) P_{\text {ave }}\left(\rho_{\text {ave }}\right) d \rho_{\text {ave }} .
$$

$P_{\mathrm{PDF}, \text { local }}\left(\rho \mid \rho_{\text {ave }}\right)$ is the conditional probability distribution function for density $\rho$, given the average $\rho_{\text {ave }}$. We assume a local PDF from supersonic turbulence:

$$
P_{\mathrm{PDF}, \text { local }}^{\prime}(\rho)=\left(2 \pi D^{2}\right)^{-1 / 2} e^{-0.5\left(\ln \left(\rho / \rho_{\mathrm{pk}}\right) / D\right)^{2}},
$$

where $P^{\prime}$ indicates the function is written per unit logarithm of the argument. The peak and average densities are related by

$$
\rho_{\mathrm{pk}}=\rho_{\mathrm{ave}} e^{-0.5 D^{2}}
$$

and the Mach number $M$ and log-normal width $D$ are related approximately by (Padoan, Nordlund \& Jones 1997)

$$
D^{2}=\ln \left(1+0.25 \mathcal{M}^{2}\right) .
$$

The average density profile is assumed to be a cored power law,

$$
\rho_{\text {ave }}(r)=\rho_{\text {edge }} \frac{r_{\text {edge }}^{\alpha}+r_{\text {core }}^{\alpha}}{r^{\alpha}+r_{\text {core }}^{\alpha}} .
$$

Then equation (26) becomes (Elmegreen 2011)

$$
P_{\mathrm{PDF}, 3 \mathrm{D}}^{\prime}(y)=\frac{3 \mathcal{C}}{\alpha(2 \pi)^{0.5}} \int_{1 / \mathcal{C}}^{1} \exp \left(-\frac{\ln ^{2}\left(y z e^{0.5 D^{2}}\right)}{2 D^{2}}\right) \frac{(z \mathcal{C}-1)^{(3-\alpha) / \alpha}}{D(\mathcal{C}-1)^{3 / \alpha}} d z,
$$

where $y=\rho / \rho_{\text {edge }}$ is the local density including turbulent fluctuations, normalized to the value at the cloud edge, $z=\rho_{\text {edge }} / \rho_{\text {ave }}(r)$ is the inverse of the average density, and $\mathcal{C}$ is the degree of central concentration,

$$
\mathcal{C}=\frac{\rho_{\text {ave }}(r=0)}{\rho_{\text {edge }}} .
$$


In the dynamical model of star formation, essentially all of the gas evolves toward higher density when it is not being pushed back by stellar pressures or sheared out by galactic rotation. The result is a delayed or resistive collapse, i.e., one filled with obstacles, but still a progression toward higher densities at some fraction of the dynamical rate. Such a model implies that the $3 \mathrm{D}$ star formation rate, $\rho_{\mathrm{SFR}}$, is proportional to the integral of the density-dependent collapse rate over the entire 3D density PDF:

$$
\rho_{\mathrm{SFR}}=\epsilon_{\mathrm{ff}} \int_{0}^{\infty} P_{\mathrm{PDF}, 3 \mathrm{D}}(\rho)\left(\rho / t_{\mathrm{ff}}\right) d \rho .
$$

There is no lower limit to the density in this expression because the low-density gas contributes very little to the integral. In practice, this lower limit is around $\rho_{\text {edge }}$ and the value of that is approximately the average midplane density. Lower-density gas tends to be warm or hot-phase and unable to join the cooler gas that is condensing from self-gravity. For numerical integrations in this sub-section, we take the lower limit of the integral equal to $0.001 \rho_{\text {edge }}$ and the upper limit $10^{6} \rho_{\text {edge }}$.

The KS relation uses the SFR surface density, for which we should integrate $\rho_{\mathrm{SFR}}$ over the line of sight through the galaxy. For the moment we write this as

$$
\Sigma_{\mathrm{SFR}}=2 H \rho_{\mathrm{SFR}}
$$

The total gas column density is

$$
\Sigma_{\text {gas }}=2 H \int_{0}^{\infty} P_{\mathrm{PDF}, 3 \mathrm{D}}(\rho) \rho d \rho
$$

and the molecular column density is

$$
\Sigma_{\mathrm{mol}}=2 H \int_{\rho_{\mathrm{mol}}}^{\infty} P_{\mathrm{PDF}, 3 \mathrm{D}}(\rho) \rho d \rho,
$$

where we use a fixed lower limit to the density in the region of the interstellar medium where the molecules appear. This is considered to be an effective minimum density for emission (Glover \& Clark 2012; Shirley 2015; Jiménez-Donaire et al. 2017; Leroy et al. 2017a) and is taken to have a universal value independent of star formation rate and interstellar structure. In Section 4 we also consider a local column density threshold for the appearance of molecules.

Figure 11 plots $\Sigma_{\text {SFR }}$ versus $\Sigma_{\text {gas }}$ as a blue curve and $\Sigma_{\text {SFR }}$ versus $\Sigma_{\text {mol }}$ as a red curve assuming $\alpha=2$. These quantities come from the PDF integrals without conversion to physical units and are shown to illustrate the slopes. Each curve is a sequence of increasing $\rho_{\text {edge }}$, which tracks the density variation in the interstellar medium. We assume the scale 
height $H$ is constant for these curves. The effect of increasing $\rho_{\text {edge }}$ is shown in Figure 2 , which plots the 3D density PDFs from equation (31) assuming a core-to-edge density contrast $\mathcal{C}=10^{5}$. Each curve uses a different $\rho_{\text {edge }}$, increasing from $7.9 \times 10^{-3}$ to $3.2 \times 10^{2}$ in equation (31) as the curves move to the right. The vertical line in Figure 2 is the fixed value of $\rho_{\mathrm{mol}}$. As mentioned above, both $\Sigma_{\mathrm{SFR}}$ and $\Sigma_{\text {gas }}$ come from integrals under the whole curve, but $\Sigma_{\text {mol }}$ comes only from the integral of the part of the curve to the right of the vertical line.

The assumption of a constant scale height in Figure 1 makes the total-gas relation (blue curve) have a power of 1.5 like the $3 \mathrm{D}$ relation because $\Sigma_{\text {gas }}$ is proportional to $\rho_{\text {edge }}$. The molecular gas relation is different with a slope of unity. This difference is because of the constant density, $\rho_{\text {mol }}$, used as a lower limit in the PDF integral for the red curve. At high surface density, the molecular slope also becomes 1.5 because most of the disk has a density above the molecular excitation density. In terms of the PDFs, this means that most of the PDF has shifted to the right of the $\rho_{\text {mol }}$ in Figure 2 so the total integral over the PDF from $\rho=0$ to $\rho=\infty$ is about the same as the integral above $\rho_{\text {mol }}$.

This shift from a linear law to a 1.5 law is consistent with observation of the KS law which show a steepening slope from $\sim 1$ in the main parts of spiral galaxy disks to $\sim 1.5$ in the inner regions where the molecular fraction is high (Kennicutt 1998). Leroy et al. (2017b) found a steepening in the relation for $\mathrm{CO}$ toward the inner part of M51. The high $\Sigma_{\mathrm{CO}}$ of starburst galaxies in Gao \& Solomon (2004) produced a steep molecular relation too. A steep relation was observed for HCN at high star formation rates in ULIRGS (Graciá-Carpio et al. 2008). Kepley et al (2014) observed a high star formation rate per unit HCN luminosity in the center of the starburst M82. The decrease in the molecular depletion time with decreasing radius in galaxies found by Utomo et al. (2017) may be the same effect.

The cyan curve in Figure 1] shows $\Sigma_{\mathrm{SFR}}$ versus $f_{\mathrm{mol}} \Sigma_{\text {gas }}$, which is the molecular part of the gas determined with fraction $f_{\text {mol }}$ from timing (Paper I). Using the PDF, this fraction becomes the ratio of average times in the molecular and total-gas phases,

$$
f_{\mathrm{mol}}=\frac{\int_{\rho_{\mathrm{mol}}}^{\infty} P_{\mathrm{PDF}, 3 \mathrm{D}}(G \rho)^{-0.5} d \rho / \int_{\rho_{\mathrm{mol}}}^{\infty} P_{\mathrm{PDF}, 3 \mathrm{D}} d \rho}{\int_{0}^{\infty} P_{\mathrm{PDF}, 3 \mathrm{D}}(G \rho)^{-0.5} d \rho / \int_{0}^{\infty} P_{\mathrm{PDF}, 3 \mathrm{D}} d \rho}
$$

this is the quantity used in the figure. The cyan curve is parallel to the red curve throughout Figure 1, meaning that the molecular fraction obtained from integrating the PDF over densities exceeding $\rho_{\text {mol }}$ is the same as the fraction of the time that the total gas spends in the molecular phase.

Semenov et al. (2017) obtain the linear molecular law in a numerical simulation because opacity provides a lower limit to the densities of molecular regions and feedback from star formation provides an upper limit. With the resulting molecular density in a narrow range, 
equations (36) and (37) apply and $t_{\mathrm{ff}, \mathrm{mol}}$ is nearly constant. Similarly, numerical simulations by Padoan et al. (2016b) of molecular cloud formation in a turbulent medium find best agreement with observations when the molecular fraction increases suddenly at a characteristic density, effectively giving most of the molecular gas approximately that density.

In gas-dominated regions of a galaxy, such as the outer parts of spirals and most of dwarf irregulars where $\sigma$ is also nearly constant, the gas scale height is determined by self-gravity, $H=\sigma^{2} / \pi G \Sigma_{\text {gas }}$, as mentioned above. If $\rho_{\text {edge }}$ is approximately the mid-plane density, then $H=\sigma /\left(2 \pi G \rho_{\text {edge }}\right)^{0.5}$, which means that at the edge of the self-gravitating structures,

$$
\rho_{\text {edge }} \sim \frac{\pi G \Sigma_{\mathrm{gas}}^{2}}{2 \sigma^{2}} .
$$

Figure 3 shows the KS relations in this case, using equation (38) for $\rho_{\text {edge }}$ in equation (32) for $\mathcal{C}$, along with equation (31) for the PDF. Now $\Sigma_{\mathrm{SFR}} \propto \Sigma_{\text {gas }}^{2}$ as in equation (12), but $\Sigma_{\mathrm{SFR}}$ is still proportional to the first power of $\Sigma_{\text {mol }}$ except in the high- $\Sigma_{\text {gas }}$, molecular-dominated regions, where the squared-law appears for molecules too.

Such a squared-law for molecules has not been observed in galaxies yet because usually the molecular-dominated interstellar regions are stellar-dominated in mass. Only individual molecular clouds have shown a squared molecular KS law so far, as discussed in Section 2.5. There may be applications of the squared molecular law in high-redshift galaxies which might have both gas dominating the stars and molecules dominating the gas (for a review of the high-redshift KS relation, see Tacconi et al. 2013).

\subsection{A Simple Model Illustrating the Slopes of the KS Relation in a Dynamical Interstellar Medium}

Considering the complexity of equation (31), a simpler derivation could make the results more intuitive. Here we consider just the power-law part of the PDF to illustrate the various slopes of the KS relations and the effect of widespread density gradients of the type $\rho \propto r^{-\alpha}$. As mentioned above, this gradient translates to a power-law column density PDF with a slope of $-1-2 /(\alpha-1)$ when plotted in linear intervals of column density, and $-2 /(\alpha-1)$ when plotted in logarithmic intervals. For 3D density, these PDFs have slopes of $-1-3 / \alpha$ and $-3 / \alpha$, respectively. We now use this power law PDF to derive the various KS slopes shown in Figures 1 and 3 .

To be specific, we write the normalized 3D PDF for $\alpha=2$ as

$$
P_{3 \mathrm{D}, \mathrm{PDF}} d \rho=1.5 \rho_{\text {edge }}^{1.5} \rho^{-2.5} d \rho
$$


between $\rho=\rho_{\text {edge }}$ and $\rho=\rho_{\max }>>\rho_{\text {edge }}$; the average density out to the edge is $\bar{\rho}\left(r_{\text {edge }}\right)=$ $3 \rho_{\text {edge }}$. We define the scale height based on the average quantities, indicated by a bar over the symbol: $H=\sigma(2 \pi G \bar{\rho})^{-0.5}=\sigma^{2}\left(\pi G \bar{\Sigma}_{\text {gas }}\right)^{-1}$ where $\bar{\Sigma}_{\text {gas }}=2 H \bar{\rho}$. Then

$$
\begin{aligned}
& \Sigma_{\mathrm{SFR}}=2 H \epsilon_{\mathrm{ff}} \int_{\rho_{\text {edge }}}^{\rho_{\max }} P_{3 \mathrm{D}, \mathrm{PDF}}(\rho)\left(\rho / t_{\mathrm{ff}}\right) d \rho=3 H \epsilon_{\mathrm{ff}}(32 G /[3 \pi])^{0.5} \rho_{\text {edge }}^{1.5} \int_{\rho_{\text {edge }}}^{\rho_{\max }} \rho^{-1} d \rho \\
& =(4 G /[9 \pi H])^{0.5} \epsilon_{\mathrm{ff}} \bar{\Sigma}_{\text {gas }}^{1.5} \ln \left(\rho_{\text {max }} / \rho_{\text {edge }}\right) \quad(\text { constant } \mathrm{H}) \\
& =(2 / 3) \epsilon_{\text {ff }}\left(G \bar{\Sigma}_{\text {gas }}^{2} / \sigma\right) \ln \left(\rho_{\max } / \rho_{\text {edge }}\right) \quad(\text { constant } \sigma) \text {. }
\end{aligned}
$$

The first result is for a region in a galaxy where the scale height is constant, and the second result is for a region where the velocity dispersion is constant. These expressions have the same forms as equations (3) and (12), respectively, with additional weak dependencies on $\rho_{\text {edge }}$.

The average surface density comes from an integral as in equation (35),

$$
\bar{\Sigma}_{\text {gas }}=2 H \int_{\rho_{\text {edge }}}^{\rho_{\text {max }}} P_{\mathrm{PDF}, 3 \mathrm{D}}(\rho) \rho d \rho=\frac{3 \sigma \rho_{\text {edge }}}{(2 \pi G)^{0.5}} \times \int_{\rho_{\text {edge }}}^{\rho_{\text {max }}} \rho^{-1.5} d \rho=\frac{6 \sigma \rho_{\text {edge }}^{0.5}}{(2 \pi G)^{0.5}}
$$

The average molecular surface density is:

$$
\begin{aligned}
\bar{\Sigma}_{\mathrm{mol}}=2 H \int_{\rho_{\mathrm{mol}}}^{\rho_{\max }} P_{\mathrm{PDF}, 3 \mathrm{D}} & (\rho) \rho d \rho=\frac{3 \sigma \rho_{\mathrm{edge}}}{(2 \pi G)^{0.5}} \times \int_{\rho_{\mathrm{mol}}}^{\rho_{\mathrm{max}}} \rho^{-1.5} d \rho \\
& =\left(\frac{1}{6 H \rho_{\mathrm{mol}}}\right)^{0.5} \bar{\Sigma}_{\text {gas }}^{1.5}(\text { constant } \mathrm{H}) \\
& =\left(\frac{\pi G}{6 \sigma^{2} \rho_{\mathrm{mol}}}\right)^{0.5} \bar{\Sigma}_{\text {gas }}^{2}(\text { constant } \sigma) .
\end{aligned}
$$

The $\Sigma_{\text {gas }}$ dependencies for molecular column density are the same as the $\Sigma_{\text {gas }}$ dependencies for $\Sigma_{\mathrm{SFR}}$, so the two scale linearly with each other, as discussed in Section 3.1 .

The molecular fraction also follows from this simple model using the fraction of the time spent in the molecular phase (i.e., at $\rho>\rho_{\text {mol }}$ ), from equation (37),

$$
f_{\mathrm{mol}}=\frac{\int_{\rho_{\mathrm{mol}}}^{\rho_{\mathrm{max}}} P_{\mathrm{PDF}, 3 \mathrm{D}}(G \rho)^{-0.5} d \rho / \int_{\rho_{\mathrm{mol}}}^{\rho_{\text {max }}} P_{\mathrm{PDF}, 3 \mathrm{D}} d \rho}{\int_{\rho_{\mathrm{edge}}}^{\rho_{\mathrm{max}}} P_{\mathrm{PDF}, 3 \mathrm{D}}(G \rho)^{-0.5} d \rho / \int_{\rho_{\text {edge }}}^{\rho_{\max }} P_{\mathrm{PDF}, 3 \mathrm{D}} d \rho}=\left(\frac{\rho_{\mathrm{edge}}}{\rho_{\text {mol }}}\right)^{0.5} .
$$

Then $\Sigma_{\text {mol }}=f_{\text {mol }} \Sigma_{\text {gas }}$ from equations (41) and (42), and $\Sigma_{\mathrm{SFR}}=\epsilon_{\mathrm{ff}} f_{\mathrm{mol}} \Sigma_{\mathrm{gas}} / t_{\mathrm{ff} \text {,mol }}$ to within a factor of $2 / \ln \left(\rho_{\max } / \rho_{\text {edge }}\right)$. This slight inaccuracy for the simple model is the difference between integrating over $\rho^{1.5}$ in equation (40) and taking the product of the integral over $\rho$ from equation (41) and the $\rho$ part of the dynamical rate, $\rho^{0.5}$. 
The various star formation relations are shown graphically in Figure 4. The total gas relation is plotted with two blue lines. It has a slope of 1.5 where the scale height is about constant, which tends to be in the star-dominated regions at high $\Sigma_{\text {tot }}$ and high $\Sigma_{\text {mol }}$. Because $H \sim$ constant, $\sigma$ is expected to decrease with increasing galacto-centric radius approximately as an exponential with a scale length that is twice the disk scale length for the stars. The gasdominated regions are shown by a blue line at low $\Sigma_{\text {gas }}$ with a slope of 2 . In a spiral galaxy, the transition from star-dominated to gas-dominated occurs in the outer disk, so a single radial profile should show both KS-1.5 and KS-2a if it goes far enough. In a dwarf irregular galaxies, only the gas-dominated part might be present and then the total relation is KS-2a with a slope of $\sim 2$, as found by Elmegreen \& Hunter (2015). The molecular star formation relation is shown in Figure 4 by a red line. This has a slope of 1 at low-to-moderate $\Sigma_{\text {mol }}$ because of the selection effect to pick regions defined by a characteristic density, $\rho_{\text {mol }}$, which makes $t_{\mathrm{ff}}=t_{\mathrm{ff}, \mathrm{mol}}$ constant when the molecular fraction is low (e.g., Krumholz \& Thompson 2007).

On a log-log plot like Figure 4, the molecular fraction at a particular star formation rate is the difference between the logs of the molecular and total surface densities, represented by the horizontal distance between the blue and red lines. In the star-dominated regions at low-to-moderate $\Sigma_{\text {mol }}$, the molecular fraction scales with the square root of the total gas surface density. In the gas-dominated regions, it scales with the first power. These scalings are evident directly from the figure and may also be derived from equation (43): if $H=$ constant in the first case, then $\rho_{\text {edge }} \propto \Sigma_{\text {gas }}$ and $f_{\text {mol }} \propto \Sigma_{\text {gas }}^{0.5}$ (as also recognized by Heiderman et al. 2010; Krumholz et al. 2012); if $H=\sigma^{2} / \pi G \Sigma_{\text {gas }}$ in the second case, then $\rho_{\text {edge }} \propto \Sigma_{\text {gas }}^{2}$, and $f_{\text {mol }} \propto \Sigma_{\text {gas }}$.

The power law expression for the PDF is simple enough to allow us to see a problem with a SFR based on equation (5) if the power in the radial profile of density, $\alpha$, is not equal to 2 , as assumed above. For a more general case with 3D PDF power $\gamma=3 / \alpha$ on a log-log plot, the normalized PDF is $P_{\mathrm{PDF}, 3 \mathrm{D}} d \rho=\gamma \rho_{\text {edge }}^{\gamma} \rho^{-1-\gamma} d \rho$. Then the integrals in equations (40)-(42) become

$$
\begin{gathered}
\Sigma_{\mathrm{SFR}}=\left(\frac{16 G(\gamma-1)^{3}}{3 \pi H(\gamma-1.5)^{2} \gamma}\right)^{0.5} \epsilon_{\mathrm{ff}} \bar{\Sigma}_{\text {gas }}^{1.5}(\text { constant } \mathrm{H}) \\
=\left(\frac{16(\gamma-1)^{3}}{3(\gamma-1.5)^{2} \gamma}\right)^{0.5} \epsilon_{\mathrm{ff}}\left(G \bar{\Sigma}_{\text {gas }}^{2} / \sigma\right) \quad(\text { constant } \sigma) \\
\bar{\Sigma}_{\text {gas }}=\left(\frac{\gamma}{\gamma-1}\right)^{0.5}\left(\frac{2 \sigma}{(2 \pi G)^{0.5}}\right) \rho_{\text {edge }}^{0.5} \\
\bar{\Sigma}_{\text {mol }}=\left(\frac{\gamma-1}{2 \gamma H \rho_{\mathrm{mol}}}\right)^{\gamma-1} \bar{\Sigma}_{\text {gas }}^{\gamma}(\text { constant } \mathrm{H})
\end{gathered}
$$




$$
=\left(\frac{(\gamma-1) \pi G}{2 \gamma \sigma^{2} \rho_{\mathrm{mol}}}\right)^{\gamma-1} \bar{\Sigma}_{\mathrm{gas}}^{2 \gamma-1}(\text { constant } \sigma) .
$$

Now the $\Sigma_{\text {gas }}$ dependencies for molecular column density and $\Sigma_{\text {SFR }}$ are not the same if $\alpha \neq 2$, so the SFR does not scale linearly with molecules. The above equations suggest $\Sigma_{\mathrm{SFR}} \propto \Sigma_{\mathrm{mol}}^{1.5 / \gamma}$, which is sub-linear for shallow cloud profiles, $\alpha<2$, and the corresponding $\gamma>1.5$. Note that this $\gamma$ is the slope of the 3D density PDF for log intervals of density, which is not observed directly. The slope of the PDF for surface density, which is directly observed, is related to this $\gamma$ by

$$
\gamma_{2 \mathrm{D}}=\frac{2 \gamma}{3-\gamma}
$$

Thus $\gamma>1.5$ for the slope of the 3D density PDF on a log-log plot corresponds to $\gamma_{2 \mathrm{D}}>2$ for the slope of the observed surface density PDF on a log-log plot.

This sublinear behavior with $\alpha<2$ and $\gamma_{2 \mathrm{D}}>2$ is only for the molecular KS relationship; the KS slope is still 1.5 for total gas regardless of $\alpha$ and $\gamma_{2 \mathrm{D}}$ (eg. 44). The sublinear molecular relation implies that at high gas surface density, an increasing fraction of the observed molecules are in the form of diffuse clouds, i.e., not strongly self-gravitating, and therefore not contributing to the SFR at the full dynamical rate. Also, it means that at low gas surface density, there is star formation in a phase of gas that is not revealed by that particular molecular emission, in dark molecular gas or atomic gas. We discuss the sublinear KS relation more in the next section.

\section{The KS Relation with a column density threshold for molecules}

Observations suggest that the KS relation for HCN sometimes becomes sublinear with too little emission for the SFR at high surface densities (Chen et al. 2015; Bigiel et al. 2016; Gowardhan et al. 2017), and sometimes becomes sublinear with excess HCN for the SFR at low surface densities (Usero et al. 2015; Kauffmann et al. 2017a). Sublinear molecular emission like this was discussed in the previous section as a possible result of a shallow density profile inside molecular clouds $(\alpha<2)$ in a large-scale survey, where the projected $\mathrm{PDF}$ for the cloud population is steep $\left(\gamma_{2 \mathrm{D}}>2\right)$.

A sublinear slope results when there are molecular emission regions that are not collapsing into stars, because that moves the observations to the right in the KS diagram without moving them up. Radiative heating of the HCN by local stars can do this (Shimajiri et al. 2017), as that increases the $\mathrm{HCN}$ luminosity for a given $\mathrm{H}_{2}$ mass and star formation rate. $\mathrm{CO}$ observations in local galaxies have also been plotted with a sublinear slope (Shetty et al. 2014), with the interpretation that much of the CO is in a diffuse, non-gravitating phase. 
Diffuse CO could be responsible for a decrease in the star formation efficiency with increasing molecular mass for clouds in the Milky Way (Ochsendorf et al. 2017). HCN also contains a diffuse component in the central parts of galaxies, where the pressure is high (Helfer \& Blitz 1997; Kauffmann et al. 2017b). Molecules that require a certain column density for selfshielding or extinction, such as $\mathrm{H}_{2}$ or $\mathrm{CO}$ (Pineda et al. 2008), should have a sub-linear KS relation when this column density is less than the threshold for strong self-gravity, which depends on pressure (Eq. 24).

A column density threshold for molecule visibility has about the same effect as decreasing the value of $\rho_{\text {mol }}$ for an increase in $\Sigma_{\text {gas }}$. Decreasing $\rho_{\text {mol }}$ extends the integral in equation (36) to include a larger part of the PDF in molecular form, increasing $\Sigma_{\text {mol }}$ without changing the integral in equations (33i) and (40) that control $\Sigma_{\mathrm{SFR}}$.

In the present model with average density variations like $\rho=\rho_{\text {edge }} r_{\text {edge }}^{2} / r^{2}$, we can write a characteristic column density $\Sigma_{\text {edge }}=\rho_{\text {edge }} r_{\text {edge }}$. For a self-gravitating medium at pressure $P, \Sigma_{\text {edge }} \sim \Sigma_{\text {Threshold }}$ for large-scale $r_{\text {edge }}$. Also with this density gradient, a cloud's mass out to the edge is $M_{\text {edge }}=4 \pi \rho_{\text {edge }} r_{\text {edge }}^{3}$. Combining these quantities gives

$$
r_{\text {edge }}=\left(\frac{M_{\text {edge }}}{4 \pi \Sigma_{\text {edge }}}\right)^{0.5} .
$$

Now consider an effective critical surface density for the appearance of a particular molecule, $\Sigma_{\mathrm{c}}$. Because in general for this density gradient, $\Sigma(r)=\rho_{\text {edge }} r_{\text {edge }}^{2} / r$, the critical radius in a cloud that has this surface density is

$$
r_{\mathrm{c}}=\frac{\rho_{\text {edge }} r_{\text {edge }}^{2}}{\Sigma_{\mathrm{c}}}=\left(\frac{\Sigma_{\text {edge }}}{\Sigma_{\mathrm{c}}}\right)\left(\frac{M_{\text {edge }}}{4 \pi \Sigma_{\text {edge }}}\right)^{0.5} .
$$

Putting this radius into the $\rho(r)$ density law gives the corresponding effective critical density,

$$
\rho_{\mathrm{c}}=\frac{\Sigma_{\text {edge }} r_{\text {edge }}}{r_{\mathrm{c}}^{2}}=\Sigma_{\mathrm{c}}^{2}\left(\frac{4 \pi}{\Sigma_{\text {edge }} M_{\text {edge }}}\right)^{0.5} .
$$

This equation states that a critical column density has a corresponding critical density that depends on cloud mass and decreases slowly with increasing pressure (through Eq. 24).

Figure 5 shows the KS relation for a hypothetical molecule that has a constant density threshold at low $\Sigma_{\text {gas }}$, and a constant surface density threshold at high $\Sigma_{\text {gas }}$. The 1.5 slope is still present for the total gas and for the molecular emission at high surface density, the linear slope is present for the molecule at low surface density, but now a sub-linear slope is present at moderate-to-high surface density where there is a surface density threshold for the molecule. Two sample cases are indicated by the split in the red and cyan lines. These 
curves are solutions to equations (33)-(36) using equation (31) as in figure 11, but now the lower limit to equation (36) is $\rho_{\mathrm{c}}$ from equation (50). $\rho_{\mathrm{c}}$ is taken to be 100 for the same range

of $\rho_{\text {edge }}$ as in Figure 2, but for $\rho_{\text {edge }}>1, \rho_{\mathrm{c}}$ in equation (36) is replaced by $\rho_{\mathrm{c}}=100 \rho_{\text {edge }}^{-0.5}$ in one case (slope 0.86 line) and $100 \rho_{\text {edge }}^{-2}$ in the other case (slope 0.64 line).

These hypothetical examples illustrate how molecular tracers can have a sublinear slope in the KS relation if the molecular gas is in a diffuse, non-self-gravitating state. Such a state tends to occur when the cloud column density is less than the threshold value given by equation (24). If the column density of a molecule exceeds a first threshold for the existence or appearance of the molecule, but not the second threshold given by self-gravity (Eq. 24), then it should present a sub-linear slope on the KS relation. Another way to say this is that molecular clouds of a certain column density (for detection) that are self-gravitating at low pressure will not be self-gravitating at high pressure.

At high column density, i.e., one that is above both the detection and the self-gravity thresholds, the KS relation for total gas mass should be recovered, i.e., with a slope of 1.5 if the galaxy thickness is about constant. This is shown in Figure 5 as well. The sub-linear to super-linear transition is reminiscent of observations by Leroy et al. (2017b), who find this for $\mathrm{CO}$ in M51.

Mok et al. (2016) found that the molecular fraction, as determined from $\mathrm{CO}(3-2)$ and HI emission, for gas in Virgo spiral galaxies is higher than in group galaxies. They also found that the molecular emission is high in Virgo compared to the star formation rate. These observations suggest there is an excess of non-star-forming, or diffuse, CO gas in Virgo spirals compared to group spirals. According to equations (24) and (50), a high interstellar pressure would do this by lowering the density at which CO is observed, thereby making proportionally more $\mathrm{CO}$, and by increasing the column density at which clouds become selfgravitating, thereby limiting the fraction of the interstellar medium that forms stars. High pressure in Virgo cluster spirals is expected because of the high intergalactic pressure from hot gas and the ram pressure from galaxy motions (Mok et al. 2017).

\section{The appearance of threshold densities and column densities for star formation}

\subsection{Observations and Models with Thresholds}

In typical regions that have been observed, stars tend to form where the column density exceeds a certain value corresponding to $\sim 8 \mathrm{mag}$ of visual extinction in the solar neighborhood (Onishi et al. 1998; Goldsmith et al. 2008; Lada et al. 2010; Heiderman et al. 2010; 
Froebrich \& Rowles 2010; Kainulainen et al. 2011; Schneider et al. 2013; Evans et al. 2014), and they also form at a rate approximately proportional to the mass of dense gas as traced by HCN (Gao \& Solomon 2004; Wu et al. 2005, 2010; Zhang et al. 2014; Shimaiiri et al. 2017), CS (Wang et al. 2011), far-infrared emission (Vutisalchavakul et al. 2014, 2016), and extinction (Lada et al. 2010; Heiderman et al. 2010; Lada et al. 2012; Evans et al. 2014).

These observations have led to the idea that star formation occurs at densities or surface densities higher than some threshold value, and that it occurs at a nearly constant rate above that value, regardless of density. This section discusses the first point, the appearance of a threshold density, using PDFs for collapse timing. The next section applies the result to dense gas surveys as an illustration of the second point.

Threshold densities have been part of star formation theory for a long time. They are assumed in numerical simulations to prevent excessively short timesteps that slow the evolution down. For example, the EAGLE simulation (Schaye et al. 2015) has a threshold density that varies with metallicity from galaxy to galaxy as $0.1(Z / 0.002)^{-0.64} \mathrm{~cm}^{-3}$, and Illustris (Vogelsberger et al. 2014) has a constant threshold, $0.13 \mathrm{~cm}^{-3}$. Hu et al. (2016) simulate dIrrs with a constant threshold of $100 \mathrm{~cm}^{-3}$. Hopkins, et al. (2017) note that the threshold value does not matter in a simulation as long as it is high enough to avoid the essential physics of interstellar collapse; this is the same point as in the present paper, where there is no physical density threshold separating star-forming gas from non-star-forming gas (see also Saitoh et al. 2008).

Analytical derivations of the star formation rate (e.g., Elmegreen 2002; Krumholz \& McKee 2005; Hennebelle \& Chabrier 2011; Padoan \& Nordlund 2011) also assume threshold densities to get the efficiency of star formation correctly when integrating over the PDF above this density. For example, Padoan et al. (2017) define a critical density as the external density of a critical Bonnor-Ebert sphere that fits in the postshock layer of a supersonically turbulent gas. Hennebelle \& Chabrier (2011) choose a critical density at which fluctuations smaller than a fixed fraction of the cloud size can fragment. Various models like this are reviewed in Federrath \& Klessen (2012) and Padoan et al. (2014).

The dynamical model discussed here does not need a threshold density for star formation because it reproduces the large-scale star formation properties of galaxies by assuming the entire interstellar medium is evolving at some fixed fraction of the dynamical rate, with no transition below and above any particular density. The model only has characteristic densities or surface densities for the detection of certain molecules, but not for the star formation process itself. The same model was applied on the molecular cloud scale by Parmentier \& Pfalzner (2013) to study the formation of bound clusters. Parmentier (2016) also considered there is no physical density threshold for star formation, but interpret the 
appearance of one as the result of combining local sources with a steep KS relation inside individual clouds and distant sources with a linear relation from poor angular resolution.

Burkert \& Hartmann (2013) and Lada et al. (2013) explain the appearance of a threshold as the result of a decreasing cloud area at higher surface density, with no actual threshold-like change for the KS relation inside molecular clouds.

Threshold-free models do not deny that certain densities play an important role in regulating the various stages of star formation. At high density, magnetic fields should decouple fast from the gas (Goodman et al. 1998; Elmegreen 2007b) and turbulent motions become subsonic causing turbulent fragmentation to stop (Padoan 1995; Vazquez-Semadeni, et al. 2003; Krumholz \& McKee 2005). The present model implies two other things instead, that the rate limiting step for star formation on a large scale is the free fall time at the lowest density, and that stars of a certain young age tend to appear where $t_{\mathrm{ff}}$ is about this age. Also, the lack of a bump or leveling off of the density PDF in star forming regions prior to the power law part from collapse implies that there is no bottleneck at some physical threshold between no star formation and star formation.

\subsection{Star Formation in Dense Gas as a Probabilistic Effect rather than a Threshold}

Here we show that the appearance of a threshold density for star formation could be a manifestation of the dynamical process itself in the sense that very young objects tend to appear at a density where the dynamical time is comparable to their age. Star formation tracers with very young ages tend to show up at densities where the dynamical time is short.

This proposed correlation between stellar age and dynamical time follows from two concepts. First, stars drift a distance proportional to their age and relative speed, so young stars are still near their birth sites, and, second, interstellar gas tends to be hierarchically clumped, so if a star forms in a certain dense region, then there is likely to be another dense region nearby (e.g., Gouliermis et al. 2017; Grasha et al. 2017). Putting these together means that young stars tend to be found near high gas densities, and slightly older stars, which have drifted further from their birth sites, tend to be near gas that has a more average, i.e., lower, density. Stars sufficiently old will have drifted past many molecular clouds in their lifetimes and they will have lost any correlation with the density of their birth.

The implications of this proposed age-density correlation may be illustrated by the timedependent collapse of a spherical cloud core, whose radius evolution is given by (Spitzer 1978)

$$
\frac{d^{2} r}{d t^{2}}=-\frac{G M\left(r_{\text {init }}\right)}{r^{2}}
$$


The solution is

$$
r=r_{\text {init }} \cos ^{2} \beta
$$

where

$$
\beta+0.5 \sin 2 \beta=\frac{\pi}{2} \frac{t}{t_{\mathrm{ff}}}
$$

is solved iteratively. Here, $t$ is the time when $r=r_{\text {init }}$ and $t_{\mathrm{ff}}$ is the free fall time at the density when the collapse begins. The solution gives the radius as a function of time and therefore the density as a function of time.

If stars form hierarchically (i.e., together) on a dynamical timescale, then the youngest stars will still be near gas where other stars are forming on about the same timescale. If young stars of a particular type have an average age $T_{\text {star }}$, then these stars are likely to be in a region where the time before other star formation, i.e., at the singularity of the collapse, $t-t_{\mathrm{ff}}$, is comparable to $T_{\mathrm{star}}$. The density at this time $t$ is known from the above solution, so we can plot the probability of seeing this star, which is approximately

$$
P_{\text {star }}(\rho)=\frac{T_{\text {star }}}{T_{\text {star }}+t_{\text {ff }}-t(\rho)},
$$

as a function of the instantaneous density, $\rho(t)$. This plot is shown in figure 6 for 3 stellar ages and 4 initial densities of the collapse, $1,10,10^{2}$, and $10^{3}$ in $\mathrm{cm}^{-3}$ of $\mathrm{H}_{2}$; Helium and heavy elements are included in the mass density for $t_{\mathrm{ff}}$. The different initial densities show up as different starting points for the curves in the lower left, but at the low values assumed, they have little effect on $\rho(t)$ after the start.

The curves show an increasing probability of seeing the stars with increasing density. The time at the half-probability point, in units of the starting $t_{\mathrm{ff}}$, ranges from 0.999 to 0.991 for a starting density of $1 \mathrm{H}_{2} \mathrm{~cm}^{-3}$ as $T_{\text {star }}$ increases from $3 \times 10^{4}$ yrs to $3 \times 10^{5}$ yrs, it ranges from 0.997 to 0.972 at a starting density of $10,0.991$ to 0.910 at a starting density of 100 , and 0.972 to 0.716 at a starting density of $1000 \mathrm{H}_{2} \mathrm{~cm}^{-3}$. The stars appear at the very last few percent of the collapse time after the density has become high, regardless of when the collapse started. Such timing would give the appearance of a threshold density when in fact the collapse is continuous with no physical threshold separating stability from collapse. Similar curves result from other collapse solutions (e.g., Huff \& Stahler 2006) as long as there is a singularity in density at a certain time.

Although this is an idealized model of singular cloud core collapse, it illustrates the basic point that in a probabilistic sense, the youngest objects should appear near gas at the highest densities. They should also appear near each other because of the hierarchical structure of the gas, and those that have already formed should be near others that are just about to form. This correlation between age and density should persist even to larger scales, 
as long as the interstellar medium is in a state of resisted collapse where all phases last for some relatively constant number of dynamical times within the full cycle of cloud formation and dispersal.

\section{On the appearance of a constant star formation rate per unit dense gas mass}

The increased likelihood for young stars to appear in dense gas implies that star formation rates correlate best with the mass of dense nearby gas. Such correlations are commonly found, and they seem to contradict the dynamical model which also involves density (e.g., Evans et al. 2014). Here we show that the star formation rate should be weakly dependent on density, as observed, even in the dynamical model if dense gas is defined either by emission from molecules with a fixed characteristic density for emission, or by a high column density observed in dust emission or extinction. The first point with dense gas defined by densetracing molecules like HCN is essentially the same as that discussed in Sections 2.3 and 3 , where the density used for the free fall time is a factor of order unity times the characteristic density of the molecule's emission.

The second point follows from equation (50) when a region is defined by a threshold column density, $\Sigma_{\mathrm{c}}$. In Section 4 we used this equation to suggest that the KS relation flattens for molecular column densities that exceed a threshold for excitation but not a threshold for self-gravity, given the ambient turbulent pressure. However, this flattening of the KS relation is also the main point of the observation that the star formation rate per unit mass is constant, regardless of density. Equation (50) shows that the threshold density for observation is insensitive to the actual cloud column density or mass when there is a constant value, $\Sigma_{\mathrm{c}}$, used to define the "dense" region. Dense gas surveys that define their selection to have extinctions exceeding $8 \mathrm{mag}$ or some such value are in this category. The free fall rate in these regions scales with $\rho_{\mathrm{c}}^{0.5}$, which scales with $\Sigma_{\text {edge }}^{-0.25}$ from equation (50), which, at the threshold of self-gravity, scales with interstellar pressure, $P$, as $P^{-0.125}$ according to equation (24). Thus there is a very weak dependence on density or ambient conditions once a self-gravitating region is chosen to exceed a certain fixed column density. The appearance of a fixed star formation rate per unit dense gas mass is thus a selection effect resulting from the definition of dense gas.

Setting the threshold column density at 8 magnitudes of visual extinction, which corresponds to $\Sigma_{\text {gas,threshold }}=160 M_{\odot} \mathrm{pc}^{-2}$, we can rewrite equation (24) as

$$
P_{\text {threshold }}=8.8 \times 10^{5} k_{\mathrm{B}}\left(\frac{\Sigma_{\text {gas,threshold }}}{160 M_{\odot} \mathrm{pc}^{-2}}\right)^{2} .
$$


The corresponding average molecular density for a velocity dispersion of $\sigma_{\text {threshold }}=10$ $\mathrm{km} \mathrm{s}^{-1}$ is $n_{\mathrm{H}_{2}}=P /\left(\mu \sigma^{2}\right)=30 \mathrm{~cm}^{-3}$ for mean molecular weight $\mu$. Evidently, when the average interstellar density exceeds the equivalent of $30 \mathrm{H}_{2} \mathrm{~cm}^{-3}$ at a $10 \mathrm{~km} \mathrm{~s}^{-1}$ velocity dispersion, "dense" regions with more than 8 magnitudes of extinction start to become diffuse and should lose their ability to form stars. This is another way of saying that the virial parameter, $5 R \sigma^{2} /(G M)$ becomes large at the corresponding cloud radius $R$ and mass $M$. Evidently, at very high pressures, "dense" gas in star forming regions should not be defined by 8 magnitudes of extinction. Defining dense gas in terms HCN emission or other dense molecular tracers may still be practical, but then the linear relation follows (KS-1a) until the average interstellar density is higher than the HCN density. Gowardhan et al. (2017) recognized this problem with the standard definition of dense gas and suggested that density contrast rather than absolute density is important.

To be more quantitative, we consider the star formation rate per unit dense gas mass, which is conveniently defined in terms of the dense gas depletion time, $M_{\text {dense }}\left(d M_{\text {stars }} / d t\right)^{-1}$ for dense gas mass $M_{\text {dense }}$ and star formation rate $d M_{\text {stars }} / d t$. For surveys with HCN and high density molecular tracers, this time has been evaluated to be $\sim 56 \mathrm{Myr}$ for galaxies in Gao \& Solomon (2004) $\sim 269$ Myr for normal spiral galaxies and $\sim 37$ Myr for ULIRGS in Liu et al. (2015), $\sim 83 \mathrm{Myr}$ for local clouds and galaxies combined in Wu et al. (2005) and Retes-Romero et al. (2017), and $\sim 60$ Myr for local clouds in Heiderman et al. (2010). For surveys at high extinction or in cloud regions with high FIR emission intensities, the dense gas depletion time has been evaluated as $\sim 22$ Myr (Lada et al. 2012; Shimajiri et al. 2017) and $\sim 25$ Myr (Evans et al. 2014) for local molecular clouds. For a combination of local clouds and galaxies using both ${ }^{13} \mathrm{CO}$ and FIR emission, Vutisalchavakul et al. (2016) got $\sim 66 \mathrm{Myr}$.

These depletion time vary between $\sim 20 \mathrm{Myr}$ and $\sim 80 \mathrm{Myr}$, depending on the sources and the observational techniques. For a representative value of $\sim 50 \mathrm{Myr}$, which means

$$
\frac{d M_{\text {stars }}}{d t}=\frac{M_{\text {dense }}}{50 \mathrm{Myr}}=\epsilon_{\mathrm{ff}} M_{\text {dense }} / t_{\mathrm{ff}, \text { dense }}
$$

we require $t_{\mathrm{ff}, \text { dense }} / \epsilon_{\mathrm{ff}, \text { dense }} \sim 50 \mathrm{Myr}$. With a characteristic density for these regions of $n_{\text {dense }} \sim 3 \times 10^{4} \mathrm{~cm}^{-3}, t_{\text {dense }}=0.19 \mathrm{Myr}$ and $\epsilon_{\mathrm{ff}}=0.004$. This is about the same result as in equation (9).

Section 2.3 discussed how some observed correlations between dense gas fractions and star formation rates can be explained in an approximate fashion by the present model. A more fundamental observation is the dense gas fraction itself. For pervasive density gradients like those assumed here, $\rho \propto 1 / r^{2}$, the mass of a gas concentration increases linearly with size and the column density decreases linearly with the inverse of size. This implies that the mass 
ratio of high density to low density gas in a cloud scales inversely with the column density thresholds used to define these gases. If extinction $A_{\mathrm{V}}=2$ defines the low density part and $A_{\mathrm{V}}=8$ defines the high density part (e.g., Evans et al. 2014), then $f_{\text {dense }}=25 \%$. If dense gas is defined by line emission that is sensitive to density rather than column density, then because mass out to some cloud radius scales inversely with the square root of density, $f_{\text {dense }}$ should be the square root of the ratio of densities of the low- and high-density tracers, which might be $\sqrt{0.01} \sim 10 \%$. These fractions are higher than the observed dense gas fraction on a large scale, which is more like 4\% (Gao \& Solomon 2004; Gowardhan et al. 2017), but the large scale contains other gas not related to star formation. Still, the pervasive appearance of self-gravitating structures shown by the power-law density PDFs on galactic scales suggests that the dense gas fraction should vary only slowly with environment, such as with radius in a galaxy or from galaxy to galaxy where the average density is less than the characteristic density of the high density tracer (e.g., normal star-forming galaxies, rather than ULIRGs). In fact, Ragan et al. (2016) find $f_{\text {dense }} \sim$ constant with radius in the Milky Way even though the star formation fraction in this dense gas varies slightly.

The dense gas fraction defined by line emission, e.g., $L_{\mathrm{HCN}} / L_{\mathrm{CO}}$, can increase substantially in ULIRGS and other regions where the average density exceeds the threshold for CO emission. The reason for this was given in Section 2.3.3, i.e., the HCN luminosity increases linearly with the star formation rate when $f_{\text {mol,HCN }}<1$, and the CO luminosity increases with the $2 / 3$ power of the star formation rate when $f_{\text {mol,CO }} \sim 1$. Then the ratio of $\mathrm{HCN}$ to CO luminosity increases with the $1 / 3$ power of the star formation rate, making it seem like regions of high star formation are different with larger dense gas fractions. In fact it is only a comparison of $f_{\text {mol }}<1$ transitions with $f_{\text {mol }} \sim 1$ transitions that does this. At low star formation rates, $f_{\text {mol }}<1$ for both high and low density molecules and both scale linearly with the star formation rate, giving a constant dense gas fraction. The dense gas fraction is defined by an observable and the observable has selection effects that influence the KS relation as much as any detail of the underlying star formation law.

Now consider the collapse model from Section 5, In a region where $t_{\mathrm{ff}}<T_{\text {star }}$, such as the dense part of a molecular cloud or a starburst galaxy with a high average interstellar density, the curves in Figure 6 all lie at high probability. Figure 7 shows examples. Now the starting densities for the $T_{\text {star }}=10^{5}$ yrs curves include higher values, $10^{4} \mathrm{~cm}^{-3}, 10^{5} \mathrm{~cm}^{-3}$ and $10^{6} \mathrm{~cm}^{-3}$, representing surveys of dense-gas regions inside molecular clouds. Also, for observations of distant galaxies, only older ages for young stellar regions can be discerned because individual stars and protostars often cannot be seen by themselves. For example, $T_{\text {star }}$ might be as high as 1 Myr for the brightest young stars, or 10 Myr for stars that excite HII regions. These cases are also in Figure 7. For high interstellar densities or relatively old stars at modest densities, the probability of seeing a star associated with the gas tends to be 
high. This would lead to the impression that stars form primarily where the average density is high, with lower average densities for older stars. The spatial and temporal resolution of events on short timescales is lost when equally young stars are not distinguished.

\section{Discussion}

A physical model for star formation that is consistent with the above explanation for the KS relations is discussed here. The main assumption is that gaseous gravity is the primary driver of star formation on a scale comparable to the scale height. As is well known, interstellar gas in a disk galaxy is stabilized against gravitational collapse by rotation on large scales and pressure on small scales (Safronov 1960; Toomre 1964; Goldreich \& Lynden Bell 1965). The mass surface density, $\Sigma_{\text {gas }}$, and epicyclic frequency, $\kappa$, determine the rotationally stabilized length in the radial direction, which is the inverse Toomre wavenumber $k_{\mathrm{T}}^{-1}=$ $2 \pi G \Sigma_{\text {gas }} / \kappa^{2}$, where self-gravity balances the Coriolis force. Because a region of this size contains the mass $M_{\mathrm{T}}=\pi \Sigma_{\text {gas }} k_{\mathrm{T}}^{-2}$, it has a specific potential energy $\sigma^{2} / 2$ equal to $G M_{\mathrm{T}} k_{\mathrm{T}}$, which corresponds to a potential velocity dispersion $\sigma=2 \pi G \Sigma_{\text {gas }} / \kappa$. For conditions in the solar neighborhood, $\Sigma_{\text {gas }} \sim 10 M_{\odot} \mathrm{pc}^{-2}$ and $\kappa=0.037 \mathrm{Myr}^{-1}$, this dispersion is $\sigma=7.5 \mathrm{~km}$ $\mathrm{s}^{-1}$, much larger than the sound speed in the cool, diffuse phase of interstellar gas, which is only $\sigma_{\mathrm{s}}=0.8 \mathrm{~km} \mathrm{~s}^{-1}$ at $100 \mathrm{~K}$. The result should be gravitationally-generated motion that is supersonic, compressive, and dissipative, with a tendency to collapse and form stars.

Magnetic forces, galactic shear, and intermittent local expansions from supernovae and other stellar feedback act to resist this collapse (Kim \& Ostriker 2015; Padoan et al. 2016a; Pan et al. 2016; Ibanez-Mejia, et al. 2016; Mac Low et al. 2017; Semenov et al. 2017). Kinematic pressures like these stabilize a disk on scales smaller than the Jeans length, $k_{\mathrm{J}}^{-1}=\sigma^{2} / \pi G \Sigma$. For an adiabatic gas, conventional theory states that if the Jeans length is larger than the Toomre length, corresponding to the condition $Q^{2}=2 k_{\mathrm{T}} / k_{\mathrm{J}}>1$, then there should be stability in the radial direction of a galaxy. Also, if $Q_{\text {eff }}>1$ for $Q_{\text {eff }}^{-2} \sim$ $Q^{-2}+2\left(k_{\text {azim }} \sigma Q / \kappa\right)^{2}$, then both radial and azimuthal motions at wavenumber $k_{\text {azim }}$ are stabilized (Lau \& Bertin 1978; Bertin 1989). However, kinematic resistance at supersonic speed damps quickly (Stone et al. 1998; Mac Low et al. 1998), so the Jeans length evaluated with supersonic $\sigma$ should not be a short-term barrier to collapse on small scales (Elmegreen 2011). In that case, $Q$ is not a measure of absolute stability. For example, in low surface-brightness regions where $Q$ appears to be high, star formation still seems normal (Elmegreen \& Hunter 2015)1 Moreover, a combined $Q$ from gas and stars appears to be dominated by stellar

\footnotetext{
${ }^{1}$ Observations of disk quenching (Martig et al. 2009; Genzel et al. 2014; French et al. 2015) seem to con-
} 
mass and motions (Romeo \& Mogotsi 2017), which means that stellar spirals, when they are present, do most of the work to regulate $Q \sim 2$, not gas processes such as star-formation feedback. Stars form because self-gravity, pressure gradients, magnetic torques, and energy dissipation decouple the gas from the stars on scales less than $k_{\mathrm{T}}^{-1}$. This makes gravitationallydriven contraction of the gas inevitable for a wide range of scales (e.g., Kravtsov et al. 2003; Li et al. 2005; Ballesteros-Paredes et al. 2011; Ibanez-Mejia, et al. 2017).

The role of the background stellar disk in the star formation process could be minor as long as the gas can dissipate thermal and turbulent energy. Gas that forms new stars has to move through the background stars to come to a high density. The two-fluid instability does not include this process because it assumes that the two fluids have perturbations with the same wavelength, which is usually the large scale of spiral arms. Gas moves through stars because of pressure forces from turbulence and stellar feedback, and it decouples from stars during the gravitational swing amplifier because of magnetic forces (Elmegreen 1987; Kim \& Ostriker 2001). The point of view here is that background stars mostly affect the thickness of the gas layer through gravity, heating, and the generation of turbulence (e.g., Ostriker et al. 2010) and therefore it affects the conversion between the observed surface density and the spatial density that controls the collapse rate. This background effect is important in the main disks of spiral galaxies and possibly elsewhere, but in the outer parts of these disks or in dwarf Irregular galaxies, the gas surface density dominates the stellar surface density and although starlight continues to heat the gas, a disk flare has a larger effect on the KS relation than the thermal properties (Elmegreen \& Hunter 2015). As long as there is a cool phase with a thermal Jean length less than the Toomre length, and as long as the timescales for thermal, turbulent, and feedback processes are comparable to or shorter than the timescale for gravitational processes, gravity, as a persistent and monotonic force toward condensation, should control the rate of star formation. Then the preceding derivations of the KS relation should contain most of the relevant physics.

\section{Conclusions}

Galaxies form stars because feedback and other energy sources are unable to resist self-gravity when gas motions are supersonic and highly dissipative. The resulting collapse is not rapid, however, because that would mix the young stellar populations and erase widespread correlations between positions and ages, which suggest turbulent compression is

tradict these statements, but perhaps quenching is from other effects, such as a high supernova rate compared to the average collapse rate, or high shear (Davis et al. 2014). 
involved. The collapse is more likely resistive, with a rate proportional to the gravity rate but significantly slower. In this case, the star formation rate per unit volume is related to the gas density in a fairly simple way (Eq. 1), and the density structure in the gas may also be written simply using a power-law PDF.

With these two assumptions, the various relationships between the star formation rate and the amount of gas can be explained by a combination of resistive collapse and a selection effect for gas observations. These relationships were divided into three types: (KS-1.5) $\Sigma_{\mathrm{SFR}} \propto \Sigma_{\text {total gas }}^{1.5}$ for main galaxy disks with constant thickness; (KS-1a) $\Sigma_{\text {SFR }} \propto \Sigma_{\text {mol }}$ for the molecular part of the gas when the average density is less than the characteristic density for observation of the molecule (and KS-1.5 again when the average density exceeds this characteristic density); (KS-1b) $\Sigma_{\text {SFR }} \propto \Sigma_{\text {gas,dense }}$ and (KS-1c) $d M_{\text {star }} / d t \propto M_{\text {dense }}$ for dense gas when the selection of this gas involves an observational threshold for either density or column density; (KS-2a) $\Sigma_{\text {SFR }} \propto \Sigma_{\text {total gas }}^{2}$ for galaxies or parts of galaxies where gaseous self-gravity determines the disk thickness and the velocity dispersion is about constant (e.g., outer parts of spirals and dIrrs); and (KS-2b) $\Sigma_{\text {SFR }} \propto \Sigma_{\text {gas }}^{2}$ inside molecular clouds where the size is also determined by gaseous self-gravity.

Numerous observations were collected to illustrate these four regimes, and the assumed model for star formation was shown to agree with the observations fairly well if the efficiency per unit free fall time is always around one percent. The model ignores the details of collapse and feedback, but the general agreement with these observations lends support to the basic premise that star formation is a pervasive dynamical process on all relevant scales.

The star formation relationships were also reproduced in general form using a probability distribution function for interstellar gas that results from turbulence convolved with self-gravitational density gradients. Using these PDFs, the molecular component can be determined either from integration above the characteristic density for observation of the molecule or from averaging over the time spent in the molecular phase. These two methods are equivalent for the dynamical model (Paper I).

A column density threshold for the detection of certain molecules flattens the KS relations by increasing the proportion of diffuse (non-gravitating) gas as the average interstellar surface density increases. Such flattening has been observed for the HCN KS relationship at both low and high pressure in different situations. Cloud selection at a minimum column density (e.g., using a lower limit to the extinction or far-infrared intensity) leads to a corresponding minimum density that varies only weakly with true cloud column density or mass, or with ambient pressure in the case of self-gravitating clouds. Then the characteristic free fall time is also nearly invariant for that region and the star formation rate per unit dense gas will be about constant. Such near-constancy will be observed even if the actual free fall 
rate scales with the square root of local density.

The appearance of a threshold density for star formation was attributed to the likely association between new-born stars and nearby gas with a free fall time comparable to the star's age. The association between young stars and high densities gets even stronger as the ambient density increases or the ages of the observable stars decreases. There is no physical threshold for star formation in this model, only an apparent one resulting from the timing of collapse. When combined with the nearly invariant collapse time in gas selected by density or column density, the observation of a universal star formation rate per unit dense gas results. Such a universal specific rate has no physical basis, however, and is even contrary to the observation that stars form in self-gravitating clouds, considering that the degree of self-gravity follows from a balance between column density and ambient pressure.

We conclude that star formation can be a continuous and dynamical process, with no activity thresholds in density or column density, and no special physics or universal rates in dense gas. The observation of star formation is fraught with selection effects, however, and this gives the KS relation many different forms.

The dynamical model gives some insight into what might be different in ultra diffuse galaxies (van Dokkum et al. 2015), damped Lyman- $\alpha$ galaxies (Rafelski et al 2016), and other seemingly failed systems where star formation rates are extremely low for the amount of gas present (e.g., Filho et al. 2016; Lisenfeld et al. 2016). If the basic ingredient for the dynamical model is supersonic turbulence generated by pervasive gravitational instabilities, then either these systems are stable, perhaps because they are thick or relatively fast-rotating, or their gas motions are subsonic, perhaps because there is no cool neutral phase. Because metallicity effects do not seem to matter for the cessation of star formation (Rafelski et al 2016), or for the star-formation rates in general in the KS relation (Section 2.4), $\mathrm{H}_{2}$ formation is apparently not involved, nor is it a necessary precursor to star formation. Lack of a cool neutral phase occurs when the pressure is very low for the ambient radiation field (Elmegreen \& Parravano 1994; Wolfire et al. 2003; Schave 2004; Kanekar et al. 2011; Krumholz 2013). This would seem to be a natural state for galaxies at very low surface densities, i.e., low pressures, where there is faint background radiation from cosmological sources and an early generation of stars.

I am grateful to Dr. Ralf Klessen for discussions at an early stage of this research and to the referee for a careful reading of the manuscript. 


\section{REFERENCES}

André, P., Menshchikov, A., Bontemps, S., et al. 2010, A\&A, 518, L102

Ballesteros-Paredes, J., Hartmann, L., Vazquez-Semadeni, E., Heitsch, F., \& Zamora-Aviles, M. A. 2011, MNRAS, 411, 65

Barnes, K. L., van Zee, L., Côté, S., \& Schade, D. 2012, ApJ, 757, 64

Bertin, G., Lin, C. C., Lowe, S. A., \& Thurstans, R. P. 1989, ApJ, 338, 104

Bigiel, F., Leroy, A., Walter, F., Brinks, E., de Blok, W. J. G., Madore, B., \& Thornley, M. D. 2008, AJ, 136, 2846

Bigiel, F., Leroy, A., Walter, F., Blitz, L., Brinks, E., de Blok, W.J.G., Madore, B. 2010, AJ, 140, 1194

Bigiel F., Leroy, A. K., Walter, F., et al., 2011, ApJ, 730, L13

Bigiel, F., Leroy, A.K., Jiménez-Donaíre, M.J. 2016, ApJL, 822, L26

Bolatto, A.D., Leroy, A.K., Jameson, K., et al. 2011, ApJ, 741, 12

Bolatto, A.D., Wong, T., Utomo, D. et al. 2017, ApJ, 846, 159

Bresolin, F., \& Kennicutt, R. C. 2015, MNRAS, 454, 3664

Buat, V., Deharveng, J. M., Donas, J. 1989, A\&A, 223, 42

Burkert, A. \& Hartmann, L. 2013, ApJ, 773, 48

Calzetti, D., Wilson, G. W., Draine, B. T., et al. 2017, ApJ, 852, 106

Chen, H., Gao, Y., Braine, J., \& Gu, Q. 2015, ApJ, 810, 140

Chen, H., Braine, J., Gao, Y., Koda, J., Gu, Q. 2017, ApJ, 836, 101

Cioffi, D.F., McKee, C.F. \& Bertschinger, E. 1988, ApJ, 334, 252

Daddi, E., Elbaz, D., Walter, F., Bournaud, F., Salmi, F., Carilli, C., Dannerbauer, H., Dickinson, M., Monaco, P., \& Riechers, D. 2010, ApJ, 714, L118

Davis, T.A., Young, L.M., Crocker, A.F., et al. 2014, MNRAS, 444, 3427

Druard, C., Braine, J., Schuster, K.F., et al. 2014, A\&A, 567, A118 
Dunham, M. M., Arce, H. G., Allen, L. E., et al. 2013, AJ, 145, 94

Elmegreen, B.G., 1987, ApJ, 312, 626

Elmegreen, B.G. 1991, ApJ, 425, L73

Elmegreen, B.G. 2002, ApJ, 577, 206

Elmegreen, B.G. 2007a, in IAU Symp. 237, Triggered Star Formation in a Turbulent ISM, ed. B. G. Elmegreen and J. Palous (Cambridge: Cambridge Univ. Press), pp.384-389

Elmegreen, B.G. 2007b, ApJ, 668, 1064

Elmegreen, B.G. 2011, ApJ, 737, 10

Elmegreen, B.G. 2013, in IAU Symp. 292, Molecular Gas, Dust, and Star Formation in Galaxies, ed. T. Wong and J. Ott (Cambridge: Cambridge Univ. Press), p. 35

Elmegreen, B.G. 2015, ApJ, 814, L30 (Paper I).

Elmegreen, B.G. \& Parravano, A. 1994, ApJL, 435, L121

Elmegreen, B.G., \& Hunter, D.A. 2015, ApJ, 805, 145

Evans, N. J., II. 1999, Ann. Rev. Ast. \& Astrophys., 37, 311

Evans, N. J., II, Allen, L. E., Blake, G. A., et al. 2003, PASP, 115, 965

Evans, N.J., II., Heiderman, A., \& Vutisalchavakul, N. 2014, ApJ, 782, 114

Federrath, C., \& Klessen, R.S. 2012, ApJ, 761, 156

Federrath C. \& Klessen R. S., 2013, ApJ, 763, 51

Filho, M. E., Sánchez Almeida, J., Amorín, R., Muñoz-Tunón, C., Elmegreen, B.G., \& Elmegreen, D. M. 2016, ApJ, 820, 109

French, K.D., Yang, Y., Zabludoff, A., Narayanan, D., Shirley, Y., Walter, F., Smith, J.-D., \& Tremonti, C.A. 2015, ApJ, 801, 1

Froebrich, D., \& Rowles, J. 2010, MNRAS, 406, 1350

Gao, Y., \& Solomon, P.M. 2004, ApJ, 606, 271

Garcia-Burillo, S., Usero, A., Alonso-Herrero, A., Graciá-Carpio, J., Pereira-Santaella, M., Colina, L., Planesas, P., \& Arribas, S. 2012, A\&A, 539, A8 
Genzel, R., Tacconi, L. J., Gracia-Carpio, J. et al. 2010, MNRAS, 407, 2091

Genzel, R., Förster Schreiber, N. M., Lang, P., et al. 2014, ApJ, 785, 75

Girichidis, P., Konstandin, L., Whitworth, A.P., \& Klessen, R.S. 2014, ApJ, 781, 91

Glover, S.C.O. \& Clark, P.C. 2012, MNRAS, 421, 116

Goldreich, P., \& Lynden-Bell, D. 1965, MNRAS, 130, 97

Goldsmith, P. F., Heyer, M., Narayanan, G., et al. 2008, ApJ, 680, 428

Goodman, A. A., Barranco, J. A.,Wilner, D. J., \& Heyer, M. H. 1998, ApJ, 504, 223

Gouliermis, D.A., Elmegreen, B.G., Elmegreen, D.M. et al. 2017, MNRAS, 468, 509

Gowardhan, A., Riechers, D.A., Daddi, E., Pavesi, R., Dannerbauer, H., Carilli, C. 2017, ApJ, 838, 136

Graciá-Carpio, J., García-Burillo, S., Planesas, P., Fuente, A., \& Usero, A. 2008, A\&A, 479, 703

Grasha, K., Calzetti, D., Adamo, A. et al. 2017, ApJ, 840, 113

Greve, T.R., Bertoldi, A.F., Smail, I., Neri, R., Chapman, S.C., Blain, A.W., Ivison, R.J., Genzel, R., Omont, A., Cox, P., Tacconi, L., \& Kneib, J.-P. 2005, MNRAS, 359, 1165

Gutermuth, R.A., Pipher, J.L., Megeath, S.T., Myers, P.C., Allen, L.E., \& Allen, T.S. 2011, ApJ, 739, 84

Harvey, P.M., Fallscheer, C., Ginsburg, A. et al. 2013, ApJ, 764, 133

Heiderman, A., Evans, N. J., II, Allen, L. E., Huard, T., \& Heyer, M. 2010, ApJ, 723, 1019

Helfer, T.T., \& Blitz, L. 1997, ApJ, 478, 162

Hennebelle, P., \& Chabrier, G. 2011, ApJL, 743, L29

Heyer, M., \& Dame, T.M. 2015, ARA\&A, 53, 583

Heyer, M.H., Gutermuth, R., Urquhart, J.S., Csengari, T., Wienen, M., Leurini, S., Menten, K., \& Wyrowski, F. 2016, A\&A, 588, A29

Hopkins, P.F., Wetzel, A., Keres, D., et al. 2017, arXiv1702.06148

Hu, Chia-Yu, Naab, T., Walch, S., Glover, S., \& Clark, P. 2016, MNRAS, 458, 3528 
Huff, E.M., \& Stahler, S.W. 2006, ApJ, 644, 355

Ibáñez-Mejía, J.C., Mac Low, M.-M., Klessen, R.S., \& Baczynski, C. 2016, ApJ, 824, 41

Ibáñez-Mejía, J.C., Mac Low, M.-M., Klessen, R.S., \& Baczynski, C. 2017, ApJ, 850, 62

Jameson, K.E., Bolatto, A.D., Leroy, A.K., et al. 2016, ApJ, 825, 12

Jiménez-Donaire, M. J., Bigiel, F., Leroy, A. K., et al. 2017, MNRAS, 466, 49

Juneau, S., Narayanan, D.T., Moustakas, J., Shirley, Y.L., Bussmann, R.S., Kennicutt, R.C., Jr. \& Vanden Bout, P.A. 2008, ApJ, 707, 1217

Kainulainen, J., Beuther, H., Banerjee, R., et al. 2011, A\&A, 530, A64

Kanekar, N., Braun, R., \& Roy, N. 2011, ApJ, 737, L33

Kauffmann, J., Goldsmith, P.F., Melnick, G., Tolls, V., Guzman, A., Menten, K.M. 2017, A\&A, 605, L5

Kauffmann, J., Pillai, T. Zhang, Q., Menten, K.M., Goldsmith, P.F., Lu, X., Guzmán, A.E., \& Schmiedeke, A. 2017, A\&A, 603, A90

Kennicutt, R.C. 1989, ApJ, 344, 685

Kennicutt, R.C., Jr. 1998, ApJ, 498, 541

Kennicutt, R.C., Jr.. Calzetti, D., Walter, F., et al. 2007, ApJ, 671, 333

Kennicutt R. C., \& Evans N. J., 2012, ARA\&A, 50, 531

Kepley, A.A., Leroy, A.K., Frayer, D., Usero, A., Marvil, J., \& Walter, F 2014, ApJL, 780, L13

Kepley, A.A., Leroy, A.K., Johnson, K.E., Sandstrom, K., Chen, C.-H.R. 2016, ApJ, 828, 50

Kim, W.-T., Ostriker, E.C. 2001, ApJ, 559, 70

Kim, C.-G., Ostriker, E.C., \& Kim, W.-T. 2013, ApJ, 776, 1

Kim, C.-G. \& Ostriker, E. 2015, ApJ, 815, 67

Klessen, R. S. 2000, ApJ, 535, 869

Kravtsov, A.V. 2003, ApJ, 590, L1 
Kritsuk, A. G., Norman, M. L., \& Wagner, R. 2011,ApJL, 727, L20

Kruijssen, J. M. D. \& Longmore, S.N. 2014, MNRAS, 439, 3239

Krumholz, M. R., \& McKee, C. F. 2005, ApJ, 630, 250

Krumholz, M.R. \& Thompson, T.A. 2007, ApJ, 669, 289

Krumholz, M.R., McKee, C.F., \& Tumlinson, J. 2009, ApJ, 693, 216

Krumholz, M. R., Dekel, A., \& McKee, C. F. 2012, ApJ, 745, 69

2012,ApJ, 759, 9

Krumholz, M.R. 2013, MNRAS, 436, 2747

Lada, C.J., Lombardi, M., \& Alves, J.F. 2010, ApJ, 724, 687

Lada, C.J., Forbrich, J., Lombardi, M., \& Alves, J.F. 2012, ApJ, 745, 190

Lada, C.J., Lombardi, M., Roman-Zuniga, C., Forbrich, J., \& Alves, J.F. 2013, ApJ, 778, 133

Lada, C.J., Lewis, J.A., Lombardi, M., \& Alves, J. 2017, A\&A, 606, A100

Larson, R.B. 1969, MNRAS, 145, 405

Larson, R.B. 1981, MNRAS, 194, 809

Lau, Y. Y., \& Bertin, G. 1978, ApJ, 226, 508

Lee, E.J., Miville-Deschênes, M.-A., Murray, N.W. 2016, ApJ, 833, 229

Leroy, A.K., Walter, F., Brinks, E., Bigiel, F., de Blok, W. J. G., Madore, B., \& Thornley, M. D. 2008, AJ, 136, 2782

Leroy, A. K., Walter, F., Sandstrom, K., et al. 2013, AJ, 146, 19

Leroy, A.K., Usero, A., Schruba, A., et al. 2017a, ApJ, 835, 217

Leroy, A.K., Schinnerer, E., Hughes, A., et al. 2017, ApJ, 846, 71

Levine, E.S., Blitz, L., \& Heiles, C. 2006, ApJ, 643, 881

Li, Y., Mac Low, M.-M., \& Klessen, R.S. 2005, ApJL, 620, L19 
Lisenfeld, U., Braine, J., Duc, P. A., Boquien, M., Brinks, E., Bournaud, F., Lelli, F., \& Charmandaris, V. 2016, A\&A, 590A, 92

Liu, L., Gao, Y., \& Greve, T.R. 2015. ApJ, 805, 31

Lombardi, M., Alves, J., Lada, C.J. 2015, A\&A, 576, L1

Mac Low, M.-M., Klessen, R.S., Burkert, A., \& Smith, M.D. 1998, PhRvL, 80, 2754

Mac Low, M.-M., Burkert, A., \& Ibáñez-Mejía, J.C. 2017, ApJL, 847, L10

Madore, B. F. 1977, MNRAS, 178, 1

Martig, M., Bournaud, F., Teyssier, R., \& Dekel, A. 2009, ApJ, 707, 250

Michalowski, M. J., Gentile, G., Hjorth, J., et al. 2015, A\&A, 582, A78

Mok,A., Wilson, C.D., \& Golding, J. 2016, MNRAS, 456, 4384

Mok, A., Wilson, C.D., Knapen, J.H., Sánchez-Gallego, J.R., Brinks, E., \& Rosolowsky, E. 2017, MNRAS, 467, 4282

Molinari, S., Swinyard, B., Bally, J., et al. 2010, A\&A, 518, L100

Mueller, K. E., Shirley, Y. L., Evans, N. J., II, \& Jacobson, H. R. 2002, ApJS, 143, 469

Murante, G., Monaco, P., Borgani, S., Tornatore, L., Dolag, K., Goz, D. 2015, MNRAS, 447,178

Murray, N. 2011, ApJ, 729, 133

Myers, P.C. 2009, ApJ, 700, 1609

Myers, P.C. 2015, ApJ, 806, 226

Narayanan, D., Cox, T. J., Shirley, Y., et al. 2008, ApJ, 684, 996

Narayanan, D., Krumholz, M.R., Ostriker, E.C., \& Hernquist, L. 2012, MNRAS, 421, 3127

Nguyen-Luong, Q., Nguyen, H.V.V., Motte, F., Schneider, N., Fujii, M., Louvet, F., Hill, T., Sanhueza, P., Chibueze, J.O., Didelon, P. 2016, ApJ, 833, 23

Nordlund, A. K., \& Padoan, P. 1999, in Interstellar Turbulence, Proceedings of the second Guillermo Haro Conference, ed. J. Franco \& A. Carraminana, (Cambridge University Press), 218 
Ochsendorf, B.B., Meixner, M., Roman-Duval, J., Rahman, M., \& Evans, N.J. II, 2017, ApJ, 841,109

Olling, R.P. 1996, AJ, 112, 457

Onishi, T., Mizuno, A., Kawamura, A., Ogawa, H., \& Fukui, Y. 1998, ApJ, 502, 296

Ostriker, E.C., McKee, C.F., \& Leroy, A.K. 2010, ApJ, 721, 975

Ostriker, E.C. \& Shetty, R. 2011, ApJ, 731, 41

Padoan, Pl. 1995, MNRAS, 277, 377

Padoan P., Nordlund A., Jones B. J. T., 1997, MNRAS, 288, 145

Padoan, P., \& Nordlund, A, 2011, ApJ, 730, 40

Padoan, P., Haugbolle, T., \& Nordlund, Å. 2012, ApJ, 759, L27

Padoan, P., Federrath, C., Chabrier, G., et al. 2014, in Protostars and Planets VI, ed. H. Beuther et al. (Tucson, AZ: Univ. of Arizona Press), 77

Padoan, P., Pan, L., Haugbølle, T., \& Nordlund, Å., 2016a, ApJ, 822, 11

Padoan, P., Juvela, M., Pan, L., Haugbølle, T., \& Nordlund, A., 2016b, ApJ, 826, 140

Padoan, P., Haugbølle, T., \& Nordlund, Å, Frimann, S. 2017, ApJ, 840, 48

Pan, L., Padoan, P., Haugbølle, T., \& Nordlund, Å., 2016, ApJ, 825, 30

Parmentier, G., Kauffmann, J., Pillai, T., \& Menten, K. M. 2011, MNRAS, 416, 783

Parmentier, G. 2016, ApJ, 826, 74

Parmentier, G. 2017, ApJ, 843, 7

Parmentier, G., \& Pfalzner, S. 2013, A\&A, 549, A132

Pineda, J. E., Caselli, P., \& Goodman, A. A. 2008, ApJ, 679, 481

Ragan, S.E., Moore, T.J.T., Eden, D.J., Hoare, M.G., Elia, D. \& Molinari, S. 2016, MNRAS, 462,3123

Rafelski, M., Gardner, J.P., Fumagalli, M., Neeleman, M., Teplitz, H.I., Grogin, N., Koekemoer, A.M., \& Scarlata, C. 2016, ApJ, 825, 87 
Renaud, F., Kraljic, K., \& Bournaud, F. 2012, ApJ, 760, L16

Renaud, F., Bournaud, F., Kraljic, K., Duc, P.-A. 2014, MNRAS, 442, L33

Retes-Romero, R., Mayya, Y. D., Luna, A., Carrasco, L. 2017, ApJ, 839, 113

Romeo, A.B., \& Mogotsi, K.M. 2017, MNRAS, 469, 286

Rosales-Ortega, F. F., Sánchez, S. F., Iglesias-Páramo, J., Dáaz, A. I., Vílchez, J. M., BlandHawthorn, J., Husemann, B., \& Mast, D. 2012, ApJL, 756, L31

Roychowdhury, S., Chengalur, J. N., Begum, A., \& Karachentsev, I. D. 2009, MNRAS, 397, 1435

Roychowdhury, S., Huang, M.-L., Kauffmann, G., Wang, J., Chengalur, J.N. 2015, MNRAS, 449,3700

Safronov, V. S. 1960, Ann.Ap., 23, 979

Saitoh, T. R., Daisaka, H., Kokubo, E., et al. 2008, PASJ, 60, 667

Schaye, J. 2004, ApJ, 609, 667

Schaye, J., Crain, R. A., Bower, R. G., et al. 2015, MNRAS, 446, 521

Schinnerer, E., Meidt, S.,E., Colombo, D., et al. 2017, ApJ, 836, 62

Schneider, N. Csengeri, T., Hennemann, M. et al. 2012, A\&A, 540, L11

Schneider, N., André, Ph., Könyves, V., et al. 2013, ApJ, 766, L17

Schneider, N. Csengeri, T., Klessen, R.S., Tremblin, P., Ossenkopf, V., Peretto, N., Simon, R., Bontemps, S., \& Federrath, C. 2015a, A\&A, 578, A29

Schneider, N., Bontemps, S. Girichidis, P. et al. 2015b, MNRAS, 453, L41

Schneider, N., Ossenkopf, V., Csengeri, T., Klessen, R.S., Federrath, C., Tremblin, P. Girichidis, P., Bontemps, S., \& André, Ph. 2015, A\&A, 575, A79

Schneider, N., Bontemps, S. Motte, F., Ossenkopf, V., Klessen, R.S., Simon, R., Fechtenbaum, S., Herpin, F., Tremblin, P., Csengeri, T., Myers, P.C., Hill, T., Cunningham, M. \& Federrath, C. 2016, A\&A, 587, A74

Schruba, A., Leroy, A.K., Walter, F., Sandstrom, K., \& Rosolowsky, E. 2010, ApJ, 722, 1699 
Schruba, A., Leroy, A. K., Walter, F., et al. 2011, AJ, 142, 37

Scoville, N., Sheth, K., Aussel, H., et al., 2016, ApJ, 820, 83

Shimajiri, Y., André, Ph., Braine, J., Könyves, V., Schneider, N., Bontemps, S,; Ladjelate, B., Roy, A., Gao, Y., \& Chen, H. 2017, A\&A, 604, A74

Semenov, V.A., Kravtsov, A.V., \& Gnedin, N.Y. 2016, ApJ, 826, 200

Semenov, V.A., Kravtsov, A.V., \& Gnedin, N.Y. 2017, ApJ, 845, 133

Shetty, R., Clark, P.C., \& Klessen, R.S. 2014, MNRAS, 442, 2208

Shirley, Y. L. 2015, PASP, 127, 299

Shu, F.H. 1977, ApJ, 214, 488

Spitzer, L., Jr. 1978, Physical Processes in the Interstellar Medium, Wiley Interscience

Stone, J. M., Ostriker, E. C., \& Gammie, C. F. 1998, ApJL, 508, L99

Tacconi, L. J., Neri, R., Genzel, R. et al. 2013, ApJ, 768, 74

Teich, Y.G., McNichols, A.T.. Nims, E., et al. 2016, ApJ, 832, 85

Tenjes, P. \& Haud, U. 1991, A\&A, 251, 11

Toomre, A. 1964, ApJ, 139, 1217

Usero, A., Leroy, A.K., Walter, F., et al. 2015, AJ, 150, 115

Utomo, D., Bolatto, A.D., Wong, T., et al. 2017, ApJ, 849, 26

van Dokkum, P.G., Abraham, R., Merritt, A., Zhang, J., Geha, M., Conroy, C. 2015, ApJ, 798, L45

Vázquez-Semadeni, E. 1994, ApJ, 423, 681

Vazquez-Semadeni, E., Ballesteros-Paredes, J., \& Klessen, R. S. 2003, ApJ, 585, L131

Vázquez-Semadeni E., González, R.F., Ballesteros-Paredes, J., Gazol, A., \& Kim, J. 2008, MNRAS, 390, 769

Vogelsberger, M., Genel, S., Springel, V., et al. 2014, MNRAS, 444, 1518

Vutisalchavakul, N., Evans, N.J., II, \& Battersby, C. 2014, ApJ, 797, 77 
Vutisalchavakul, N., Evans, N.J., II, \& Heyer, M. 2016, ApJ, 831, 73

Wang, J., Zhang, Z., \& Shi, Y. 2011, MNRAS, 416, L21

Willis, S., Guzman, A., Marengo, M., et al. 2015, ApJ, 809, 87

Wolfire, M.G., McKee, C.F., Hollenbach, D., Tielens, A.G.G.M. 2003, ApJ, 587, 278

Wong, T. \& Blitz, L. 2002, ApJ, 569, 157

Wu, J., Evans, N.J., II., Gao, Y., Solomon, P.M., Shirley, Y.L., \& Vanden Bout, P.A. 2005, ApJ, 635, L173

Wu, J., Evans, II, N.J., Shirley, Y.L., \& Knez, C. 2010, ApJS, 188, 313

Zhang, Z.-Y., Gau, Y., Henkel, C., Zhao, Y., Wang, J., Menten, K.M. \& Güsten, R. 2014, ApJ, 784, L31 


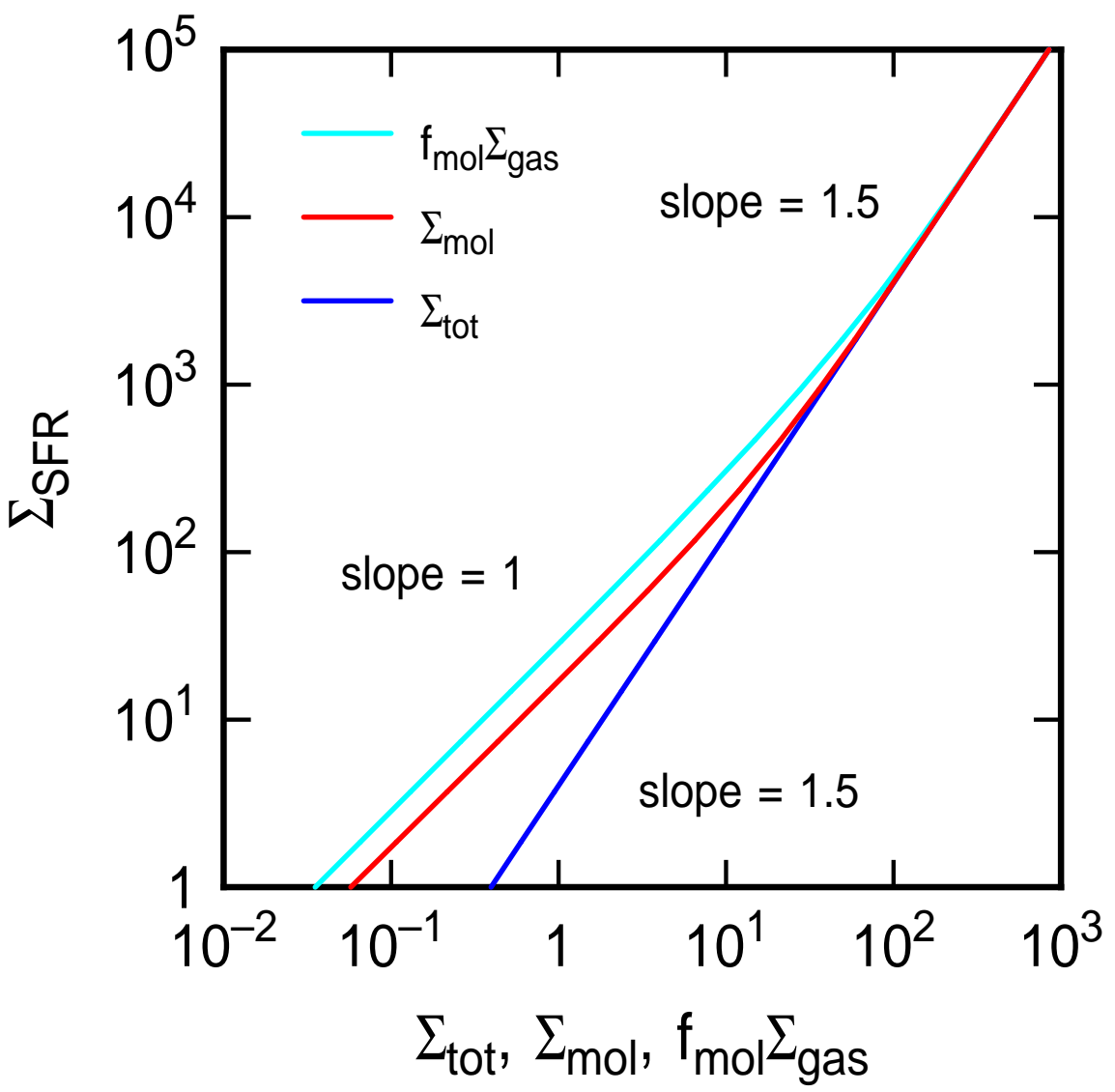

Fig. 1.- Solutions to equations (31), (33)- (37) when the average density profile inside each cloud has a slope $\alpha=2$. Two parts of the KS law in main galaxy disks are shown: (1) the 1.5 slope (blue) for all phases of gas at high gas surface density when the interstellar medium is mostly molecular and the same 1.5 slope also for any gas surface density when plotted versus total gas; (2) the linear slope for molecules (red) at low-to-intermediate surface densities when the characteristic density for molecular emission is larger than the average interstellar density. The red curve uses equation (36) directly and the cyan curve calculates the molecular fraction from timing considerations, equation (37). The offset between the red line and the cyan line is non-physical, it is the difference between integrating the PDF over $\rho^{1.5}$ and integrating it over $\rho$ with a separate multiplication by $\rho^{0.5}$. The first method is the most physically relevant and the second illustrates the importance of dynamical evolution in the molecular medium. 

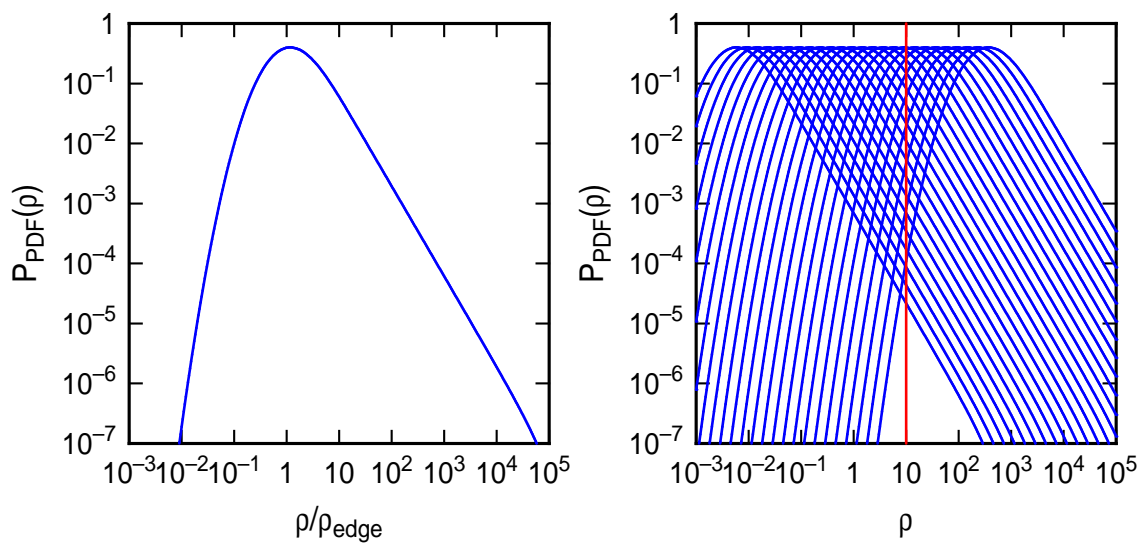

Fig. 2.- (left) The density PDF from equation (31) for a density contrast $\mathcal{C}=10^{5}$, a Mach number 2, and $\alpha=2$, normalized to unit area. (right) A sequence of PDFs with increasing $\rho_{\text {edge }}$ illustrating how the interstellar PDF moves through a constant threshold for molecular emission (red line) as $\Sigma_{\text {gas }}$ increases. This sequence corresponds to the points used to plot the curves in Figure 1 . 


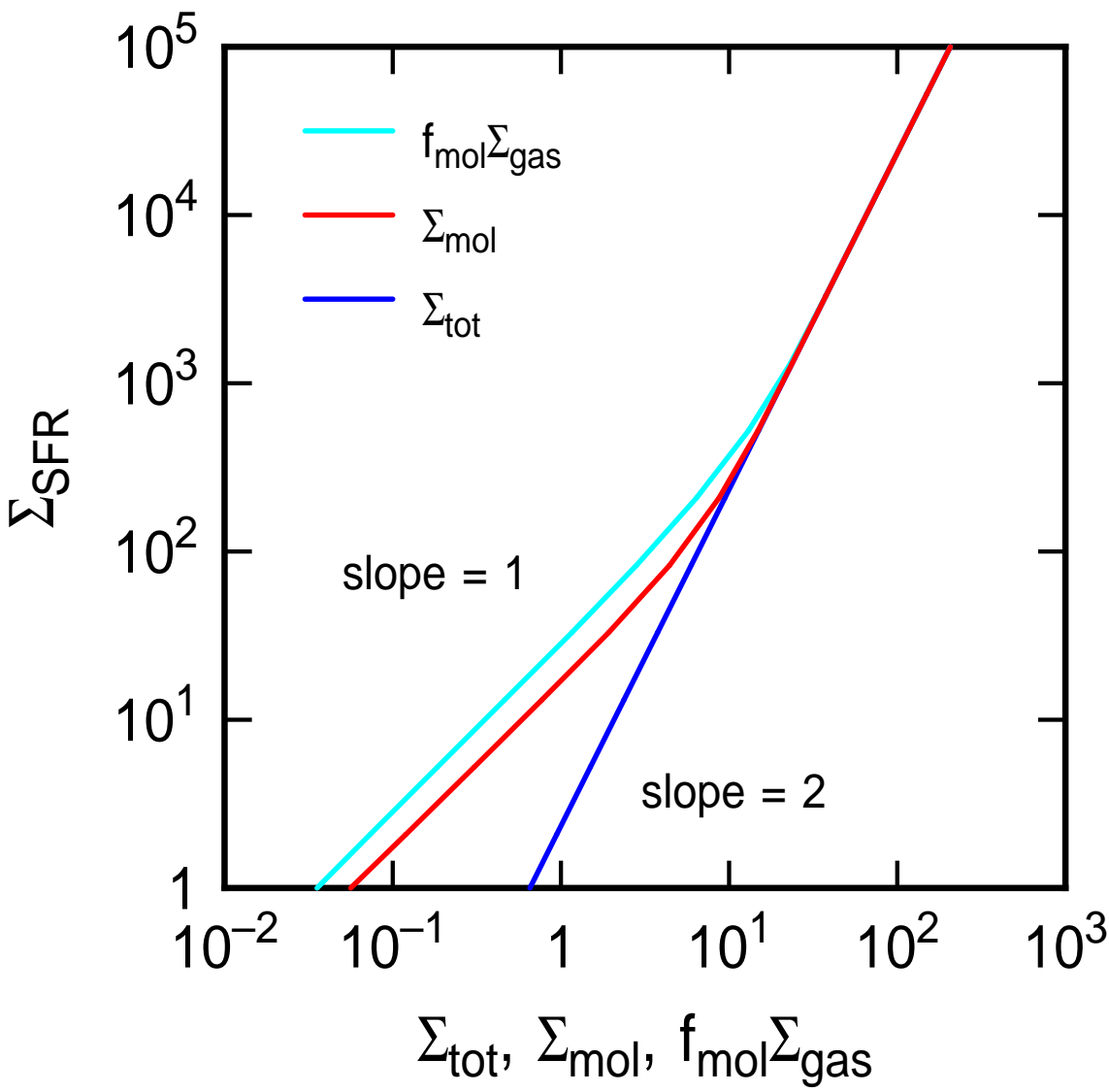

Fig. 3.- Solutions to equations (31)-(37) as in Figure 1 but for a pure-gas disk where the scale height varies inversely with the gas surface density. Now the KS relation for total gas (blue) and high-density molecular gas (blue/red) has a slope of 2, although the molecular gas (red) still has a slope of 1 at low surface density. $\alpha=2$ is assumed. 


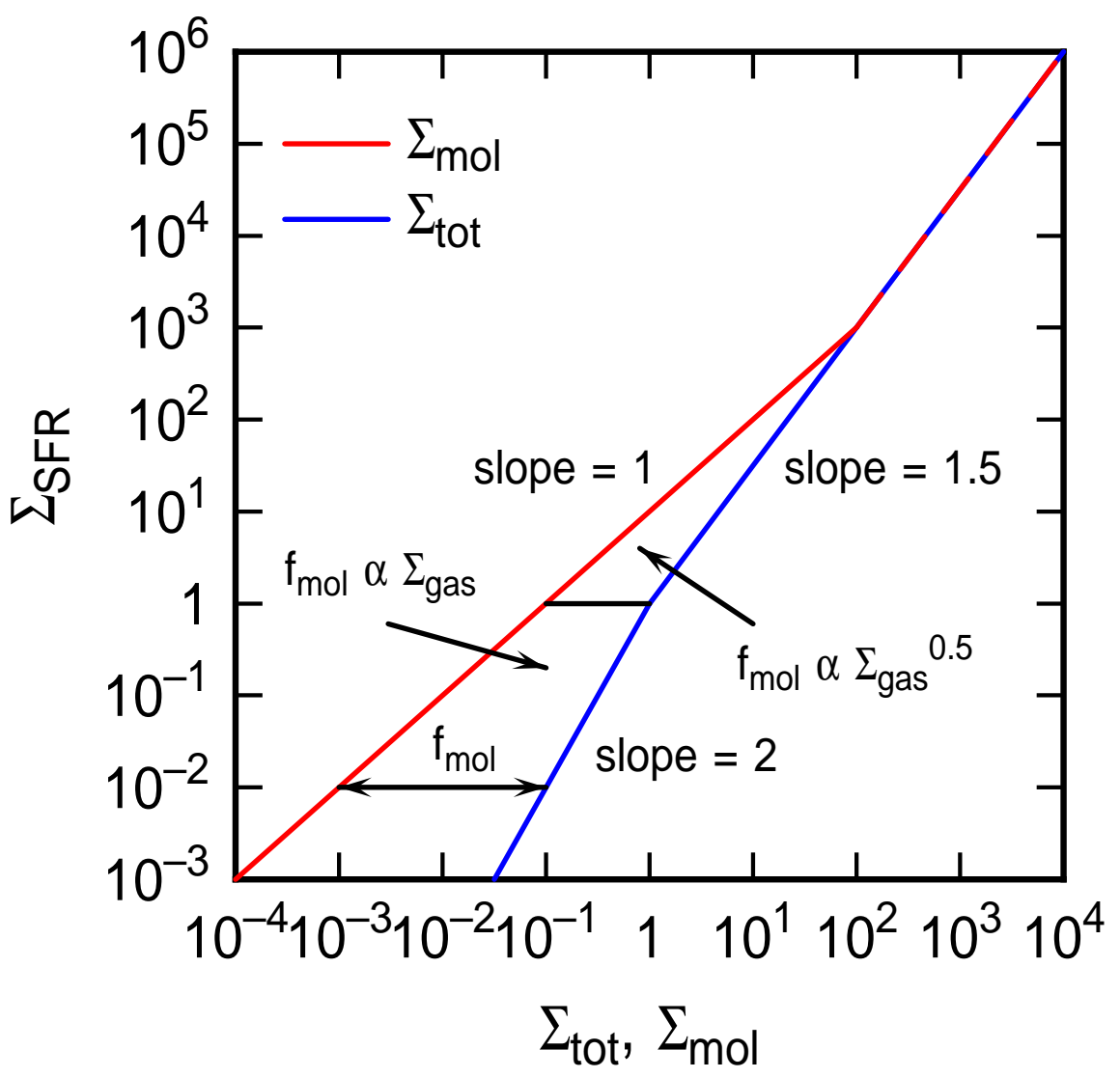

Fig. 4.- Schematic KS relation showing star formation rate surface density versus total gas (blue) and molecular gas (red) as calculated from equations (39) - (43), which assume a power-law PDF. The slope is 1.5 for all phases at high gas surface density, 1.5 for total gas at intermediate surface density, 1 for molecular gas at intermediate-to-low surface density, and 2 for total gas at low surface density. All of these relationships follow from one threedimensional star formation law, equation (11), but they are viewed with different radial variations of galaxy thickness and different selection effects. The molecular fraction is the horizontal distance between the molecular and the total-gas KS curves in this logarithmic plot. 


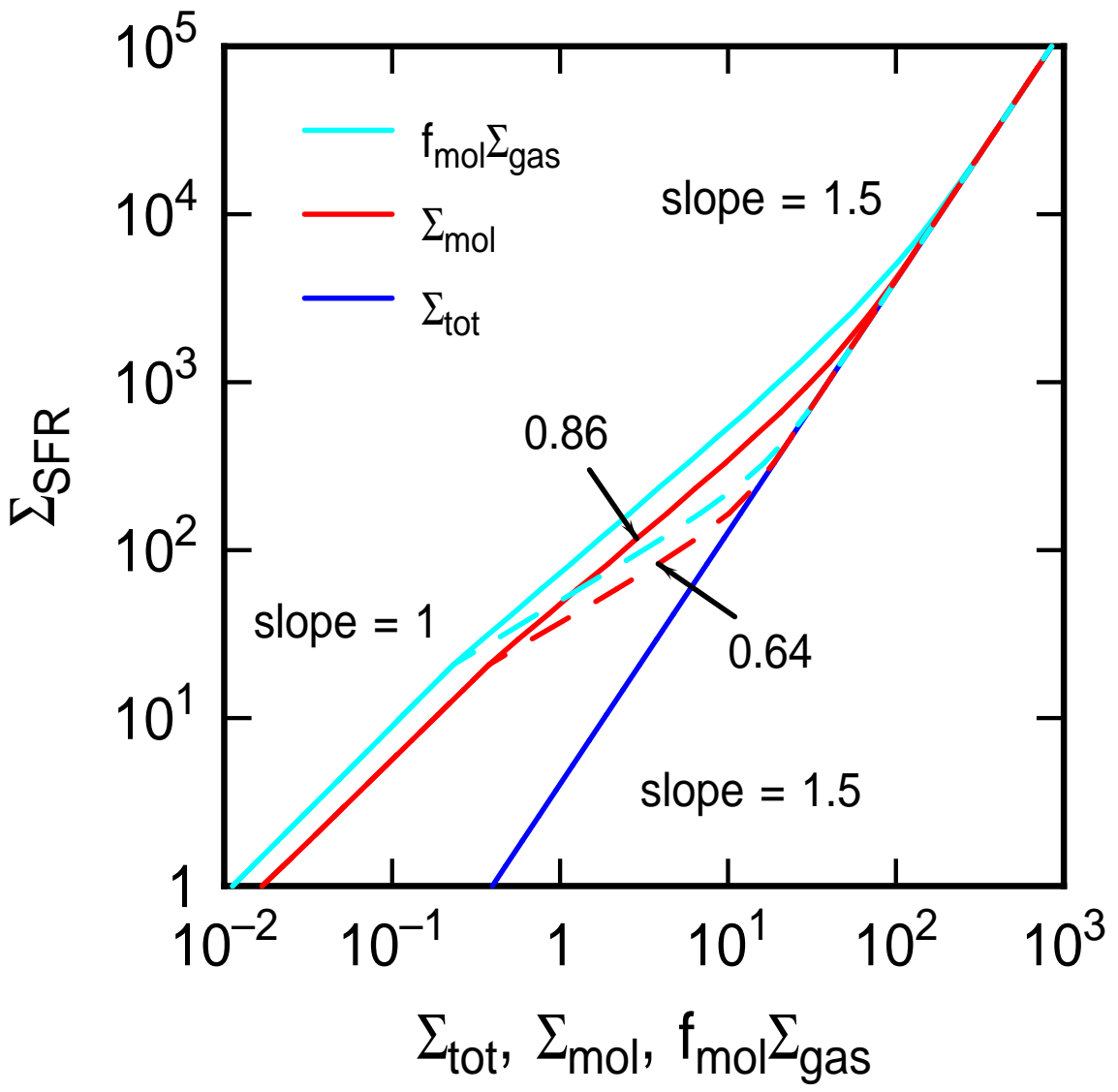

Fig. 5.- Solutions to equations (33)-(36) using equation (11) as in Figure 1, with the lower limit to equation (36) taken to be the critical density $\rho_{\mathrm{c}}$ from equation (50). This solution illustrates the flattening of the KS relation for molecules that have a threshold column density for formation or emission (which is at $\Sigma \sim 0.6$ in this figure). The split in the red and cyan curves is from two different models for how the threshold density varies with surface density (see Fig. 1). 


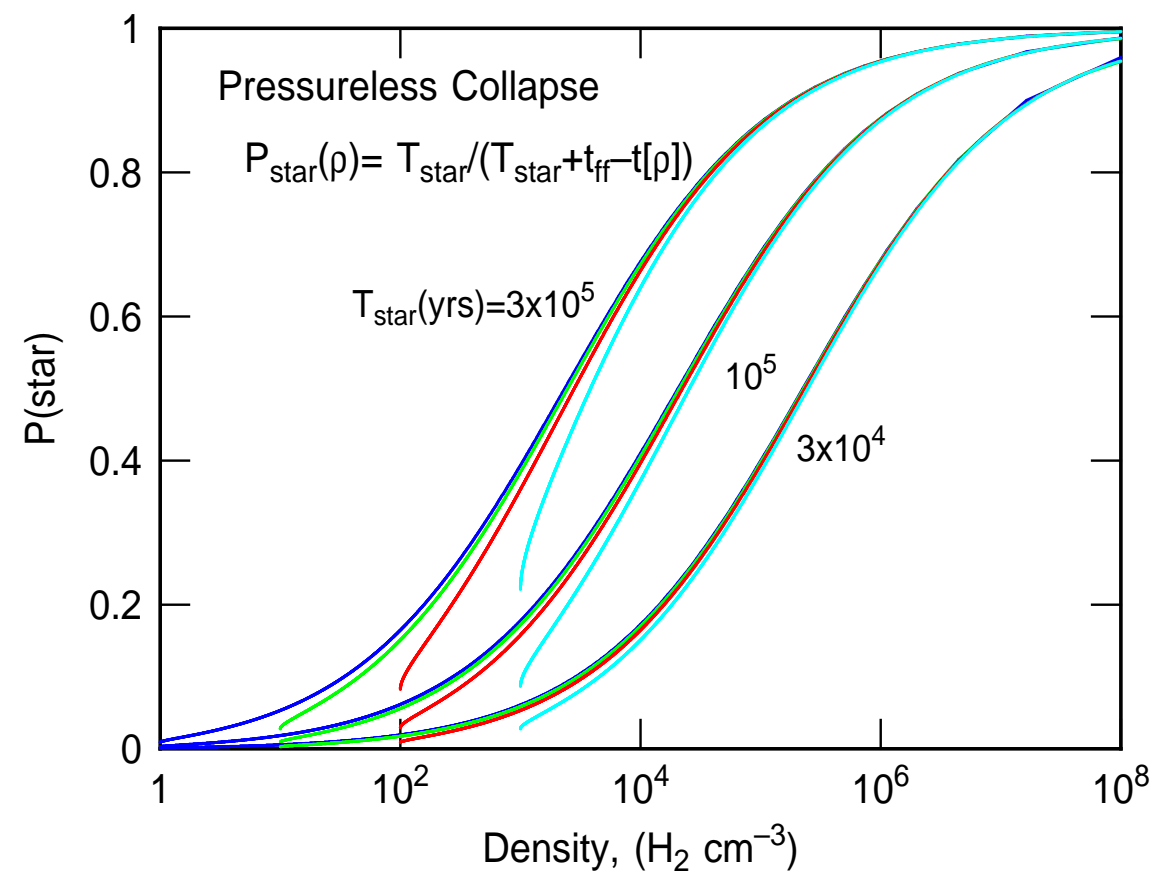

Fig. 6.- Solutions to equations (51)-(54) for the probability of observing stars with a young age, $T_{\text {star }}$, near a region of a cloud with the $\mathrm{H}_{2}$ density indicated on the abscissa. The probability increases rapidly at the density where the free fall time is comparable to the age of the star. Different curves for the same $T_{\text {star }}$ are for different starting densities in the collapsing cloud. 


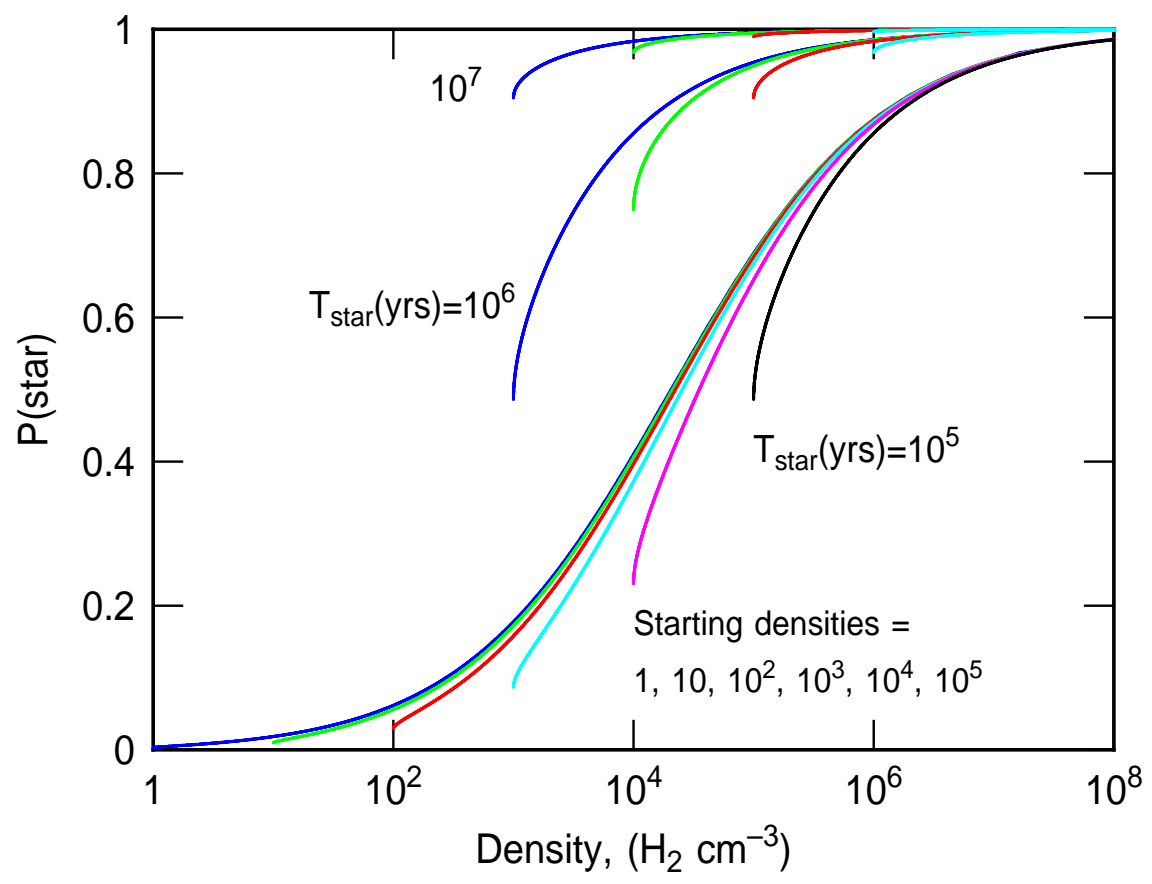

Fig. 7.- Same as Figure 6 but for a wider range of starting densities in the lower curves and for larger stellar ages in the upper curves. This figure shows that stars slightly older than the free fall time in a gas selected for observation will usually appear to be associated with that gas, giving the impression for the youngest observable stars that they tend to form where the average gas density is high. 